Supporting Information

\title{
Enforced Planar FOX-7-like Molecules: A Strategy for Thermally Stable and Insensitive $\pi$-Conjugated Energetic Materials
}

Yongxing Tang, ${ }^{*}+\star$ Wei Huang, ${ }^{\dagger}$ Gregory H. Imler, ${ }^{\S}$ Damon A. Parrish ${ }^{\S}$ and Jean’ne M.

Shreeve*,

$\dagger$ Dr. Y. Tang, Dr. W. Huang, Nanjing University of Science and Technology, Nanjing, 210094 (China)

$\ddagger$ Dr. Y. Tang, Prof. J. M. Shreeve, Department of Chemistry, University of Idaho, Moscow, Idaho, 83844-2343 USA. Fax: (+1) 208-885-5173 E-mail: jshreeve@ uidaho.edu

$\S$ Dr. G. H. Imler, Dr. D. A. Parrish, Naval Research Laboratory, 4555 Overlook Avenue, Washington, D.C. 20375 USA 
Table of contents

$\begin{array}{ll}\text { Crystal Structure Analysis } & \text { S3 }\end{array}$

Covalent Bonding Interactions

S23 


\section{Crystal Structure Analysis}

Table S1. Crystal data and structure refinement for 3 .

\begin{tabular}{|c|c|c|}
\hline Identification code & \multicolumn{2}{|l|}{3} \\
\hline CCDC number & \multicolumn{2}{|l|}{1975685} \\
\hline Empirical formula & \multicolumn{2}{|l|}{$\mathrm{C}_{3} \mathrm{H}_{6.33} \mathrm{~N}_{7} \mathrm{O}_{2.67}$} \\
\hline Formula weight & \multicolumn{2}{|l|}{183.15} \\
\hline Temperature & \multicolumn{2}{|l|}{ 293(2) K } \\
\hline Wavelength & \multicolumn{2}{|l|}{$1.54178 \AA$} \\
\hline Crystal system & \multicolumn{2}{|l|}{ Triclinic } \\
\hline Space group & \multicolumn{2}{|l|}{ P-1 } \\
\hline Unit cell dimensions & $\begin{array}{l}a=4.9771(4) \AA \\
b=13.1099(9) \AA \\
c=16.9277(11) \AA\end{array}$ & $\begin{array}{l}\alpha=101.610(4)^{\circ} . \\
\beta=91.179(5)^{\circ} . \\
\gamma=90.050(5)^{\circ} .\end{array}$ \\
\hline Volume & \multicolumn{2}{|l|}{$1081.68(14) \AA^{3}$} \\
\hline $\mathrm{Z}$ & \multicolumn{2}{|l|}{6} \\
\hline Density $\left(20^{\circ} \mathrm{C}\right)$ & \multicolumn{2}{|l|}{$1.687 \mathrm{Mg} / \mathrm{m}^{3}$} \\
\hline $\begin{array}{l}\text { Absorption coefficient } \\
F(000)\end{array}$ & \multicolumn{2}{|l|}{$1.273 \mathrm{~mm}^{-1}$} \\
\hline Crystal size & \multicolumn{2}{|c|}{$0.134 \times 0.049 \times 0.040 \mathrm{~mm}^{3}$} \\
\hline Theta range for data collection & \multicolumn{2}{|c|}{2.665 to $68.373^{\circ}$} \\
\hline Index ranges & \multicolumn{2}{|c|}{$-5<=\mathrm{h}<=5,-15<=\mathrm{k}<=12,-20<=\mathrm{l}<=20$} \\
\hline Reflections collected & \multicolumn{2}{|c|}{7269} \\
\hline Independent reflections & \multicolumn{2}{|l|}{$3470\left[\mathrm{R}_{\mathrm{int}}=0.0653\right]$} \\
\hline Completeness to theta $=67.679^{\circ}$ & \multicolumn{2}{|l|}{$88.8 \%$} \\
\hline Absorption correction & \multicolumn{2}{|c|}{ Semi-empirical from equivalents } \\
\hline Max. and min. transmission & \multicolumn{2}{|c|}{0.7531 and 0.5385} \\
\hline $\begin{array}{l}\text { Refinement method } \\
\text { Data / restraints / parameters }\end{array}$ & \multicolumn{2}{|c|}{ Full-matrix least-squares on $\mathrm{F}^{2}$} \\
\hline Goodness-of-fit on $\mathrm{F}^{2}$ & \multicolumn{2}{|l|}{1.091} \\
\hline Final $R$ indices $[I>2 \operatorname{sigma}(I)]$ & \multicolumn{2}{|c|}{$\mathrm{R}_{1}=0.0651, \mathrm{wR}_{2}=0.1872$} \\
\hline $\mathrm{R}$ indices (all data) & \multicolumn{2}{|c|}{$\mathrm{R}_{1}=0.0922, \mathrm{wR}_{2}=0.2085$} \\
\hline Largest diff. peak and hole & \multicolumn{2}{|c|}{0.278 and -0.317 e. $\AA^{-3}$} \\
\hline
\end{tabular}


Table S2. Bond lengths $[\AA]$ and angles $\left[{ }^{\circ}\right]$ for 3 .

\begin{tabular}{|c|c|c|c|}
\hline $\mathrm{O}(1)-\mathrm{N}(3)$ & $1.267(4)$ & $\mathrm{O}(2)-\mathrm{N}(3)$ & $1.266(4)$ \\
\hline $\mathrm{N}(3)-\mathrm{C}(4)$ & $1.340(5)$ & $\mathrm{C}(4)-\mathrm{C}(8)$ & $1.449(5)$ \\
\hline$C(4)-C(5)$ & $1.481(4)$ & $\mathrm{C}(5)-\mathrm{N}(7)$ & $1.292(5)$ \\
\hline $\mathrm{C}(5)-\mathrm{N}(6)$ & $1.315(5)$ & $\mathrm{N}(7)-\mathrm{H}(7 \mathrm{~A})$ & 0.8600 \\
\hline $\mathrm{N}(7)-\mathrm{H}(7 \mathrm{~B})$ & 0.8600 & $\mathrm{~N}(6)-\mathrm{H}(6 \mathrm{~A})$ & 0.8600 \\
\hline $\mathrm{N}(6)-\mathrm{H}(6 \mathrm{~B})$ & 0.8600 & $\mathrm{C}(8)-\mathrm{N}(9)$ & $1.321(5)$ \\
\hline $\mathrm{C}(8)-\mathrm{N}(12)$ & $1.343(4)$ & $\mathrm{N}(9)-\mathrm{N}(10)$ & $1.354(4)$ \\
\hline $\mathrm{N}(10)-\mathrm{N}(11)$ & $1.281(4)$ & $\mathrm{N}(11)-\mathrm{N}(12)$ & $1.343(4)$ \\
\hline $\mathrm{N}(12)-\mathrm{H}(12)$ & 0.8600 & $\mathrm{O}(13)-\mathrm{N}(15)$ & $1.257(4)$ \\
\hline $\mathrm{O}(14)-\mathrm{N}(15)$ & $1.260(4)$ & $\mathrm{N}(15)-\mathrm{C}(16)$ & $1.375(4)$ \\
\hline$C(16)-C(20)$ & $1.426(5)$ & $\mathrm{C}(16)-\mathrm{C}(17)$ & $1.463(5)$ \\
\hline $\mathrm{C}(17)-\mathrm{N}(18)$ & $1.302(5)$ & $\mathrm{C}(17)-\mathrm{N}(19)$ & $1.329(4)$ \\
\hline $\mathrm{N}(18)-\mathrm{H}(18 \mathrm{~A})$ & 0.8600 & $\mathrm{~N}(18)-\mathrm{H}(18 \mathrm{~B})$ & 0.8600 \\
\hline $\mathrm{N}(19)-\mathrm{H}(19 \mathrm{~A})$ & 0.8600 & $\mathrm{~N}(19)-\mathrm{H}(19 \mathrm{~B})$ & 0.8600 \\
\hline $\mathrm{C}(20)-\mathrm{N}(21)$ & $1.336(4)$ & $\mathrm{C}(20)-\mathrm{N}(24)$ & $1.357(4)$ \\
\hline $\mathrm{N}(21)-\mathrm{N}(22)$ & $1.347(5)$ & $\mathrm{N}(22)-\mathrm{N}(23)$ & $1.281(4)$ \\
\hline $\mathrm{N}(23)-\mathrm{N}(24)$ & $1.322(4)$ & $\mathrm{N}(24)-\mathrm{H}(24)$ & 0.8600 \\
\hline $\mathrm{O}(25)-\mathrm{N}(27)$ & $1.275(4)$ & $\mathrm{O}(26)-\mathrm{N}(27)$ & $1.245(4)$ \\
\hline $\mathrm{N}(27)-\mathrm{C}(28)$ & $1.367(4)$ & $\mathrm{C}(28)-\mathrm{C}(29)$ & $1.436(5)$ \\
\hline $\mathrm{C}(28)-\mathrm{C}(32)$ & $1.471(5)$ & $\mathrm{C}(29)-\mathrm{N}(31)$ & $1.318(5)$ \\
\hline $\mathrm{C}(29)-\mathrm{N}(30)$ & $1.326(5)$ & $\mathrm{N}(30)-\mathrm{H}(30 \mathrm{~A})$ & 0.8600 \\
\hline $\mathrm{N}(30)-\mathrm{H}(30 \mathrm{~B})$ & 0.8600 & $\mathrm{~N}(31)-\mathrm{H}(31 \mathrm{~A})$ & 0.8600 \\
\hline $\mathrm{N}(31)-\mathrm{H}(31 \mathrm{~B})$ & 0.8600 & $\mathrm{C}(32)-\mathrm{N}(36)$ & $1.325(5)$ \\
\hline $\mathrm{C}(32)-\mathrm{N}(33)$ & $1.328(4)$ & $\mathrm{N}(33)-\mathrm{N}(34)$ & $1.354(5)$ \\
\hline $\mathrm{N}(34)-\mathrm{N}(35)$ & $1.277(5)$ & $\mathrm{N}(35)-\mathrm{N}(36)$ & $1.340(4)$ \\
\hline $\mathrm{N}(36)-\mathrm{H}(36)$ & 0.8600 & & \\
\hline $\mathrm{O}(2)-\mathrm{N}(3)-\mathrm{O}(1)$ & $116.9(3)$ & $\mathrm{O}(2)-\mathrm{N}(3)-\mathrm{C}(4)$ & $120.1(3)$ \\
\hline $\mathrm{O}(1)-\mathrm{N}(3)-\mathrm{C}(4)$ & $123.0(3)$ & $\mathrm{N}(3)-\mathrm{C}(4)-\mathrm{C}(8)$ & $118.9(3)$ \\
\hline $\mathrm{N}(3)-\mathrm{C}(4)-\mathrm{C}(5)$ & 121.1(3) & $\mathrm{C}(8)-\mathrm{C}(4)-\mathrm{C}(5)$ & $120.1(3)$ \\
\hline $\mathrm{N}(7)-\mathrm{C}(5)-\mathrm{N}(6)$ & $118.9(3)$ & $\mathrm{N}(7)-\mathrm{C}(5)-\mathrm{C}(4)$ & $120.0(3)$ \\
\hline $\mathrm{N}(6)-\mathrm{C}(5)-\mathrm{C}(4)$ & $121.0(3)$ & $\mathrm{C}(5)-\mathrm{N}(7)-\mathrm{H}(7 \mathrm{~A})$ & 120.0 \\
\hline $\mathrm{C}(5)-\mathrm{N}(7)-\mathrm{H}(7 \mathrm{~B})$ & 120.0 & $\mathrm{H}(7 \mathrm{~A})-\mathrm{N}(7)-\mathrm{H}(7 \mathrm{~B})$ & 120.0 \\
\hline $\mathrm{C}(5)-\mathrm{N}(6)-\mathrm{H}(6 \mathrm{~A})$ & 120.0 & $\mathrm{C}(5)-\mathrm{N}(6)-\mathrm{H}(6 \mathrm{~B})$ & 120.0 \\
\hline $\mathrm{H}(6 \mathrm{~A})-\mathrm{N}(6)-\mathrm{H}(6 \mathrm{~B})$ & 120.0 & $\mathrm{~N}(9)-\mathrm{C}(8)-\mathrm{N}(12)$ & $107.2(3)$ \\
\hline $\mathrm{N}(9)-\mathrm{C}(8)-\mathrm{C}(4)$ & $124.9(3)$ & $N(12)-C(8)-C(4)$ & $127.9(3)$ \\
\hline $\mathrm{C}(8)-\mathrm{N}(9)-\mathrm{N}(10)$ & $106.4(3)$ & $\mathrm{N}(11)-\mathrm{N}(10)-\mathrm{N}(9)$ & $110.9(3)$ \\
\hline $\mathrm{N}(10)-\mathrm{N}(11)-\mathrm{N}(12)$ & $106.5(3)$ & $\mathrm{C}(8)-\mathrm{N}(12)-\mathrm{N}(11)$ & $108.9(3)$ \\
\hline $\mathrm{C}(8)-\mathrm{N}(12)-\mathrm{H}(12)$ & 125.5 & $\mathrm{~N}(11)-\mathrm{N}(12)-\mathrm{H}(12)$ & 125.5 \\
\hline $\mathrm{O}(13)-\mathrm{N}(15)-\mathrm{O}(14)$ & $118.5(3)$ & $\mathrm{O}(13)-\mathrm{N}(15)-\mathrm{C}(16)$ & $122.2(3)$ \\
\hline $\mathrm{O}(14)-\mathrm{N}(15)-\mathrm{C}(16)$ & $119.2(3)$ & $\mathrm{N}(15)-\mathrm{C}(16)-\mathrm{C}(20)$ & $118.4(3)$ \\
\hline$N(15)-C(16)-C(17)$ & $119.4(3)$ & $C(20)-C(16)-C(17)$ & $122.3(3)$ \\
\hline $\mathrm{N}(18)-\mathrm{C}(17)-\mathrm{N}(19)$ & $119.0(3)$ & $\mathrm{N}(18)-\mathrm{C}(17)-\mathrm{C}(16)$ & $123.1(3)$ \\
\hline $\mathrm{N}(19)-\mathrm{C}(17)-\mathrm{C}(16)$ & $117.9(3)$ & $\mathrm{C}(17)-\mathrm{N}(18)-\mathrm{H}(18 \mathrm{~A})$ & 120.0 \\
\hline $\mathrm{C}(17)-\mathrm{N}(18)-\mathrm{H}(18 \mathrm{~B})$ & 120.0 & $\mathrm{H}(18 \mathrm{~A})-\mathrm{N}(18)-\mathrm{H}(18 \mathrm{~B})$ & 120.0 \\
\hline $\mathrm{C}(17)-\mathrm{N}(19)-\mathrm{H}(19 \mathrm{~A})$ & 120.0 & $\mathrm{C}(17)-\mathrm{N}(19)-\mathrm{H}(19 \mathrm{~B})$ & 120.0 \\
\hline $\mathrm{H}(19 \mathrm{~A})-\mathrm{N}(19)-\mathrm{H}(19 \mathrm{~B})$ & 120.0 & $\mathrm{~N}(21)-\mathrm{C}(20)-\mathrm{N}(24)$ & $105.3(3)$ \\
\hline$N(21)-C(20)-C(16)$ & $125.2(3)$ & $\mathrm{N}(24)-\mathrm{C}(20)-\mathrm{C}(16)$ & $129.5(3)$ \\
\hline $\mathrm{C}(20)-\mathrm{N}(21)-\mathrm{N}(22)$ & $107.3(3)$ & $\mathrm{N}(23)-\mathrm{N}(22)-\mathrm{N}(21)$ & $110.4(3)$ \\
\hline
\end{tabular}




\begin{tabular}{lllr}
$\mathrm{N}(22)-\mathrm{N}(23)-\mathrm{N}(24)$ & $107.2(3)$ & $\mathrm{N}(23)-\mathrm{N}(24)-\mathrm{C}(20)$ & $109.8(3)$ \\
$\mathrm{N}(23)-\mathrm{N}(24)-\mathrm{H}(24)$ & 125.1 & $\mathrm{C}(20)-\mathrm{N}(24)-\mathrm{H}(24)$ & 125.1 \\
$\mathrm{O}(26)-\mathrm{N}(27)-\mathrm{O}(25)$ & $118.7(3)$ & $\mathrm{O}(26)-\mathrm{N}(27)-\mathrm{C}(28)$ & $120.7(3)$ \\
$\mathrm{O}(25)-\mathrm{N}(27)-\mathrm{C}(28)$ & $120.6(3)$ & $\mathrm{N}(27)-\mathrm{C}(28)-\mathrm{C}(29)$ & $122.2(3)$ \\
$\mathrm{N}(27)-\mathrm{C}(28)-\mathrm{C}(32)$ & $116.4(3)$ & $\mathrm{C}(29)-\mathrm{C}(28)-\mathrm{C}(32)$ & $121.3(3)$ \\
$\mathrm{N}(31)-\mathrm{C}(29)-\mathrm{N}(30)$ & $117.9(3)$ & $\mathrm{N}(31)-\mathrm{C}(29)-\mathrm{C}(28)$ & $120.3(3)$ \\
$\mathrm{N}(30)-\mathrm{C}(29)-\mathrm{C}(28)$ & $121.8(3)$ & $\mathrm{C}(29)-\mathrm{N}(30)-\mathrm{H}(30 \mathrm{~A})$ & 120.0 \\
$\mathrm{C}(29)-\mathrm{N}(30)-\mathrm{H}(30 \mathrm{~B})$ & 120.0 & $\mathrm{H}(30 \mathrm{~A})-\mathrm{N}(30)-\mathrm{H}(30 \mathrm{~B})$ & 120.0 \\
$\mathrm{C}(29)-\mathrm{N}(31)-\mathrm{H}(31 \mathrm{~A})$ & 120.0 & $\mathrm{C}(29)-\mathrm{N}(31)-\mathrm{H}(31 \mathrm{~B})$ & 120.0 \\
$\mathrm{H}(31 \mathrm{~A})-\mathrm{N}(31)-\mathrm{H}(31 \mathrm{~B})$ & 120.0 & $\mathrm{~N}(36)-\mathrm{C}(32)-\mathrm{N}(33)$ & $107.4(3)$ \\
$\mathrm{N}(36)-\mathrm{C}(32)-\mathrm{C}(28)$ & $129.2(3)$ & $\mathrm{N}(33)-\mathrm{C}(32)-\mathrm{C}(28)$ & $123.4(3)$ \\
$\mathrm{C}(32)-\mathrm{N}(33)-\mathrm{N}(34)$ & $105.9(3)$ & $\mathrm{N}(35)-\mathrm{N}(34)-\mathrm{N}(33)$ & $110.8(3)$ \\
$\mathrm{N}(34)-\mathrm{N}(35)-\mathrm{N}(36)$ & $106.5(3)$ & $\mathrm{C}(32)-\mathrm{N}(36)-\mathrm{N}(35)$ & $109.3(3)$ \\
$\mathrm{C}(32)-\mathrm{N}(36)-\mathrm{H}(36)$ & 125.3 & $\mathrm{~N}(35)-\mathrm{N}(36)-\mathrm{H}(36)$ & 125.3 \\
\hline
\end{tabular}


Table S3. Torsion angles $\left[{ }^{\circ}\right]$ for 3 .

\begin{tabular}{|c|c|c|c|}
\hline $\mathrm{O}(2)-\mathrm{N}(3)-\mathrm{C}(4)-\mathrm{C}(8)$ & $0.5(5)$ & $\mathrm{O}(1)-\mathrm{N}(3)-\mathrm{C}(4)-\mathrm{C}(8)$ & $-178.7(4)$ \\
\hline $\mathrm{O}(2)-\mathrm{N}(3)-\mathrm{C}(4)-\mathrm{C}(5)$ & $179.4(3)$ & $\mathrm{O}(1)-\mathrm{N}(3)-\mathrm{C}(4)-\mathrm{C}(5)$ & $0.1(6)$ \\
\hline $\mathrm{N}(3)-\mathrm{C}(4)-\mathrm{C}(5)-\mathrm{N}(7)$ & $-178.9(3)$ & $\mathrm{C}(8)-\mathrm{C}(4)-\mathrm{C}(5)-\mathrm{N}(7)$ & $-0.1(5)$ \\
\hline $\mathrm{N}(3)-\mathrm{C}(4)-\mathrm{C}(5)-\mathrm{N}(6)$ & $2.8(5)$ & $\mathrm{C}(8)-\mathrm{C}(4)-\mathrm{C}(5)-\mathrm{N}(6)$ & $-178.5(3)$ \\
\hline $\mathrm{N}(3)-\mathrm{C}(4)-\mathrm{C}(8)-\mathrm{N}(9)$ & $179.3(3)$ & $\mathrm{C}(5)-\mathrm{C}(4)-\mathrm{C}(8)-\mathrm{N}(9)$ & $0.4(5)$ \\
\hline $\mathrm{N}(3)-\mathrm{C}(4)-\mathrm{C}(8)-\mathrm{N}(12)$ & $-1.1(5)$ & $\mathrm{C}(5)-\mathrm{C}(4)-\mathrm{C}(8)-\mathrm{N}(12)$ & $-179.9(3)$ \\
\hline $\mathrm{N}(12)-\mathrm{C}(8)-\mathrm{N}(9)-\mathrm{N}(10)$ & $0.4(4)$ & $\mathrm{C}(4)-\mathrm{C}(8)-\mathrm{N}(9)-\mathrm{N}(10)$ & $-179.8(3)$ \\
\hline $\mathrm{C}(8)-\mathrm{N}(9)-\mathrm{N}(10)-\mathrm{N}(11)$ & $0.3(4)$ & $\mathrm{N}(9)-\mathrm{N}(10)-\mathrm{N}(11)-\mathrm{N}(12)$ & $-0.9(4)$ \\
\hline $\mathrm{N}(9)-\mathrm{C}(8)-\mathrm{N}(12)-\mathrm{N}(11)$ & $-1.0(4)$ & $\mathrm{C}(4)-\mathrm{C}(8)-\mathrm{N}(12)-\mathrm{N}(11)$ & $179.3(3)$ \\
\hline $\mathrm{N}(10)-\mathrm{N}(11)-\mathrm{N}(12)-\mathrm{C}(8)$ & $1.1(4)$ & $\mathrm{O}(13)-\mathrm{N}(15)-\mathrm{C}(16)-\mathrm{C}(20)$ & $-179.6(3)$ \\
\hline $\mathrm{O}(14)-\mathrm{N}(15)-\mathrm{C}(16)-\mathrm{C}(20)$ & $-0.8(5)$ & $\mathrm{O}(13)-\mathrm{N}(15)-\mathrm{C}(16)-\mathrm{C}(17)$ & $1.7(5)$ \\
\hline $\mathrm{O}(14)-\mathrm{N}(15)-\mathrm{C}(16)-\mathrm{C}(17)$ & $-179.5(3)$ & $\mathrm{N}(15)-\mathrm{C}(16)-\mathrm{C}(17)-\mathrm{N}(18)$ & $1.0(5)$ \\
\hline $\mathrm{C}(20)-\mathrm{C}(16)-\mathrm{C}(17)-\mathrm{N}(18)$ & $-177.7(3)$ & $\mathrm{N}(15)-\mathrm{C}(16)-\mathrm{C}(17)-\mathrm{N}(19)$ & $-178.7(3)$ \\
\hline $\mathrm{C}(20)-\mathrm{C}(16)-\mathrm{C}(17)-\mathrm{N}(19)$ & $2.6(5)$ & $\mathrm{N}(15)-\mathrm{C}(16)-\mathrm{C}(20)-\mathrm{N}(21)$ & $-176.9(3)$ \\
\hline $\mathrm{C}(17)-\mathrm{C}(16)-\mathrm{C}(20)-\mathrm{N}(21)$ & $1.8(5)$ & $\mathrm{N}(15)-\mathrm{C}(16)-\mathrm{C}(20)-\mathrm{N}(24)$ & $3.2(5)$ \\
\hline $\mathrm{C}(17)-\mathrm{C}(16)-\mathrm{C}(20)-\mathrm{N}(24)$ & $-178.1(3)$ & $\mathrm{N}(24)-\mathrm{C}(20)-\mathrm{N}(21)-\mathrm{N}(22)$ & $-0.4(4)$ \\
\hline $\mathrm{C}(16)-\mathrm{C}(20)-\mathrm{N}(21)-\mathrm{N}(22)$ & $179.7(3)$ & $\mathrm{C}(20)-\mathrm{N}(21)-\mathrm{N}(22)-\mathrm{N}(23)$ & $-0.7(4)$ \\
\hline $\mathrm{N}(21)-\mathrm{N}(22)-\mathrm{N}(23)-\mathrm{N}(24)$ & $1.6(4)$ & $\mathrm{N}(22)-\mathrm{N}(23)-\mathrm{N}(24)-\mathrm{C}(20)$ & $-1.8(4)$ \\
\hline $\mathrm{N}(21)-\mathrm{C}(20)-\mathrm{N}(24)-\mathrm{N}(23)$ & $1.3(4)$ & $\mathrm{C}(16)-\mathrm{C}(20)-\mathrm{N}(24)-\mathrm{N}(23)$ & $-178.7(3)$ \\
\hline $\mathrm{O}(26)-\mathrm{N}(27)-\mathrm{C}(28)-\mathrm{C}(29)$ & $179.5(3)$ & $\mathrm{O}(25)-\mathrm{N}(27)-\mathrm{C}(28)-\mathrm{C}(29)$ & $-1.3(5)$ \\
\hline $\mathrm{O}(26)-\mathrm{N}(27)-\mathrm{C}(28)-\mathrm{C}(32)$ & $1.5(5)$ & $\mathrm{O}(25)-\mathrm{N}(27)-\mathrm{C}(28)-\mathrm{C}(32)$ & $-179.3(3)$ \\
\hline $\mathrm{N}(27)-\mathrm{C}(28)-\mathrm{C}(29)-\mathrm{N}(31)$ & $177.6(3)$ & $\mathrm{C}(32)-\mathrm{C}(28)-\mathrm{C}(29)-\mathrm{N}(31)$ & $-4.5(5)$ \\
\hline $\mathrm{N}(27)-\mathrm{C}(28)-\mathrm{C}(29)-\mathrm{N}(30)$ & $-2.0(6)$ & $\mathrm{C}(32)-\mathrm{C}(28)-\mathrm{C}(29)-\mathrm{N}(30)$ & $176.0(4)$ \\
\hline $\mathrm{N}(27)-\mathrm{C}(28)-\mathrm{C}(32)-\mathrm{N}(36)$ & $-2.6(6)$ & $\mathrm{C}(29)-\mathrm{C}(28)-\mathrm{C}(32)-\mathrm{N}(36)$ & $179.3(4)$ \\
\hline $\mathrm{N}(27)-\mathrm{C}(28)-\mathrm{C}(32)-\mathrm{N}(33)$ & $177.6(3)$ & $\mathrm{C}(29)-\mathrm{C}(28)-\mathrm{C}(32)-\mathrm{N}(33)$ & $-0.5(6)$ \\
\hline $\mathrm{N}(36)-\mathrm{C}(32)-\mathrm{N}(33)-\mathrm{N}(34)$ & $1.0(4)$ & $\mathrm{C}(28)-\mathrm{C}(32)-\mathrm{N}(33)-\mathrm{N}(34)$ & $-179.2(4)$ \\
\hline $\mathrm{C}(32)-\mathrm{N}(33)-\mathrm{N}(34)-\mathrm{N}(35)$ & $-1.0(5)$ & $\mathrm{N}(33)-\mathrm{N}(34)-\mathrm{N}(35)-\mathrm{N}(36)$ & $0.6(5)$ \\
\hline $\mathrm{N}(33)-\mathrm{C}(32)-\mathrm{N}(36)-\mathrm{N}(35)$ & $-0.6(4)$ & $\mathrm{C}(28)-\mathrm{C}(32)-\mathrm{N}(36)-\mathrm{N}(35)$ & $179.5(4)$ \\
\hline $\mathrm{N}(34)-\mathrm{N}(35)-\mathrm{N}(36)-\mathrm{C}(32)$ & $0.0(5)$ & & \\
\hline
\end{tabular}


Table S4. Hydrogen bonds for 3 [^ and ${ }^{\circ}$ ].

\begin{tabular}{lcccc}
\hline \multicolumn{1}{c}{ D-H...A } & $\mathrm{d}(\mathrm{D}-\mathrm{H})$ & $\mathrm{d}(\mathrm{H} \ldots \mathrm{A})$ & $\mathrm{d}(\mathrm{D} \ldots \mathrm{A})$ & $<(\mathrm{DHA})$ \\
\hline $\mathrm{N}(7)-\mathrm{H}(7 \mathrm{~A}) \ldots \mathrm{O}(14)$ & 0.86 & 2.16 & $3.019(4)$ & 174.4 \\
$\mathrm{~N}(7)-\mathrm{H}(7 \mathrm{~B}) \ldots \mathrm{N}(9)$ & 0.86 & 1.95 & $2.652(4)$ & 137.4 \\
$\mathrm{~N}(6)-\mathrm{H}(6 \mathrm{~A}) \ldots \mathrm{O}(13)$ & 0.86 & 2.02 & $2.880(4)$ & 176.1 \\
$\mathrm{~N}(6)-\mathrm{H}(6 \mathrm{~B}) \ldots \mathrm{O}(1)$ & 0.86 & 1.93 & $2.574(4)$ & 131.1 \\
$\mathrm{~N}(12)-\mathrm{H}(12) \ldots \mathrm{O}(2)$ & 0.86 & 2.06 & $2.565(4)$ & 117.0 \\
$\mathrm{~N}(12)-\mathrm{H}(12) \ldots \mathrm{N}(35) \# 1$ & 0.86 & 2.25 & $3.010(4)$ & 146.7 \\
$\mathrm{~N}(18)-\mathrm{H}(18 \mathrm{~A}) \ldots \mathrm{O}(25)$ & 0.86 & 2.07 & $2.925(4)$ & 176.5 \\
$\mathrm{~N}(18)-\mathrm{H}(18 \mathrm{~B}) \ldots \mathrm{O}(13)$ & 0.86 & 1.92 & $2.558(4)$ & 130.3 \\
$\mathrm{~N}(19)-\mathrm{H}(19 \mathrm{~A}) \ldots \mathrm{O}(26)$ & 0.86 & 2.14 & $2.999(4)$ & 176.9 \\
$\mathrm{~N}(19)-\mathrm{H}(19 \mathrm{~B}) \ldots \mathrm{N}(21)$ & 0.86 & 1.96 & $2.659(4)$ & 137.8 \\
$\mathrm{~N}(24)-\mathrm{H}(24) \ldots \mathrm{N}(11) \# 2$ & 0.86 & 2.31 & $3.079(4)$ & 148.5 \\
$\mathrm{~N}(24)-\mathrm{H}(24) \ldots \mathrm{O}(14)$ & 0.86 & 2.08 & $2.578(4)$ & 116.2 \\
$\mathrm{~N}(30)-\mathrm{H}(30 \mathrm{~A}) \ldots \mathrm{O}(1) \# 3$ & 0.86 & 2.03 & $2.885(4)$ & 172.5 \\
$\mathrm{~N}(30)-\mathrm{H}(30 \mathrm{~B}) \ldots \mathrm{O}(25)$ & 0.86 & 1.92 & $2.562(4)$ & 130.3 \\
$\mathrm{~N}(31)-\mathrm{H}(31 \mathrm{~A}) \ldots \mathrm{O}(2) \# 3$ & 0.86 & 2.19 & $3.044(4)$ & 174.0 \\
$\mathrm{~N}(31)-\mathrm{H}(31 \mathrm{~B}) \ldots \mathrm{N}(33)$ & 0.86 & 1.97 & $2.655(4)$ & 136.2 \\
$\mathrm{~N}(36)-\mathrm{H}(36) \ldots \mathrm{N}(23) \# 4$ & 0.86 & 2.30 & $3.055(4)$ & 146.7 \\
$\mathrm{~N}(36)-\mathrm{H}(36) \ldots \mathrm{O}(26)$ & 0.86 & 2.05 & $2.561(4)$ & 117.0 \\
& & & & \\
\hline
\end{tabular}

Symmetry transformations used to generate equivalent atoms:

$\# 1 \mathrm{x}+3, \mathrm{y}+1, \mathrm{z} \quad \# 2-\mathrm{x}+1,-\mathrm{y}+1,-\mathrm{z}+1 \quad \# 3-\mathrm{x}-1,-\mathrm{y}+1,-\mathrm{z}+2$

$\# 4-x-2,-y,-z+1$ 
Table S5. Crystal data and structure refinement for 4 .

Identification code 4

Empirical formula

CCDC number

Formula weight

$\mathrm{C}_{4} \mathrm{H}_{6} \mathrm{~N}_{6} \mathrm{O}_{2}$

Temperature

Wavelength

Crystal system

Space group

Unit cell dimensions

Volume

$\mathrm{Z}$ 1975691

170.15

293(2) K

$1.54178 \AA$

Orthorhombic

Pbca

$$
\begin{array}{ll}
a=11.3834(3) \AA & \alpha=90^{\circ} . \\
b=7.3039(2) \AA & \beta=90^{\circ} . \\
c=15.8799(4) \AA & \gamma=90^{\circ} . \\
1320.31(6) \AA 3 &
\end{array}
$$

Density $\left(20^{\circ} \mathrm{C}\right)$

Absorption coefficient

8

$1.712 \mathrm{Mg} / \mathrm{m}^{3}$

$1.218 \mathrm{~mm}^{-1}$

$\mathrm{F}(000)$

704

Crystal size

$0.389 \times 0.020 \times 0.020 \mathrm{~mm}^{3}$

Theta range for data collection

5.572 to $74.507^{\circ}$.

Index ranges

$-12<=\mathrm{h}<=14,-8<=\mathrm{k}<=7,-19<=\mathrm{l}<=15$

Reflections collected

8602

Independent reflections

Completeness to theta $=67.679^{\circ}$

$1279\left[\mathrm{R}_{\text {int }}=0.0506\right]$

$96.2 \%$

Absorption correction

Max. and min. transmission

Semi-empirical from equivalents

0.7538 and 0.6619

Refinement method

Data / restraints / parameters

Full-matrix least-squares on $\mathrm{F}^{2}$

Goodness-of-fit on $\mathrm{F}^{2}$

1279 / 0 / 110

Final $\mathrm{R}$ indices [I $>2 \operatorname{sigma}(\mathrm{I})]$

1.093

$\mathrm{R}$ indices (all data)

$\mathrm{R}_{1}=0.0440, \mathrm{w}_{2}=0.1157$

$\mathrm{R}_{1}=0.0458, \mathrm{wR}_{2}=0.1175$

Extinction coefficient

$0.0047(7)$

Largest diff. peak and hole

0.223 and -0.245 e. $\AA^{-3}$ 
Table S6. Bond lengths $[\AA]$ and angles $\left[{ }^{\circ}\right]$ for 4 .

\begin{tabular}{llll}
\hline $\mathrm{O}(1)-\mathrm{N}(3)$ & $1.2730(16)$ & $\mathrm{O}(2)-\mathrm{N}(3)$ & $1.2616(16)$ \\
$\mathrm{N}(3)-\mathrm{C}(4)$ & $1.3620(16)$ & $\mathrm{C}(4)-\mathrm{C}(5)$ & $1.4588(18)$ \\
$\mathrm{C}(4)-\mathrm{C}(8)$ & $1.4593(19)$ & $\mathrm{C}(5)-\mathrm{N}(6)$ & $1.3141(18)$ \\
$\mathrm{C}(5)-\mathrm{N}(7)$ & $1.3168(18)$ & $\mathrm{N}(6)-\mathrm{H}(6 \mathrm{~A})$ & 0.8600 \\
$\mathrm{~N}(6)-\mathrm{H}(6 \mathrm{~B})$ & 0.8600 & $\mathrm{~N}(7)-\mathrm{H}(7 \mathrm{~A})$ & 0.8600 \\
$\mathrm{~N}(7)-\mathrm{H}(7 \mathrm{~B})$ & 0.8600 & $\mathrm{C}(8)-\mathrm{N}(12)$ & $1.3374(17)$ \\
$\mathrm{C}(8)-\mathrm{N}(9)$ & $1.3402(17)$ & $\mathrm{N}(9)-\mathrm{N}(10)$ & $1.3571(18)$ \\
$\mathrm{N}(9)-\mathrm{H}(9)$ & 0.8600 & $\mathrm{~N}(10)-\mathrm{C}(11)$ & $1.306(2)$ \\
$\mathrm{C}(11)-\mathrm{N}(12)$ & $1.3538(18)$ & & 0.9300 \\
& & $\mathrm{O}(2)-\mathrm{N}(3)-\mathrm{C}(4)$ & $120.29(12)$ \\
$\mathrm{O}(2)-\mathrm{N}(3)-\mathrm{O}(1)$ & $117.80(11)$ & $\mathrm{N}(3)-\mathrm{C}(4)-\mathrm{C}(5)$ & $120.42(12)$ \\
$\mathrm{O}(1)-\mathrm{N}(3)-\mathrm{C}(4)$ & $121.91(11)$ & $\mathrm{C}(5)-\mathrm{C}(4)-\mathrm{C}(8)$ & $121.24(11)$ \\
$\mathrm{N}(3)-\mathrm{C}(4)-\mathrm{C}(8)$ & $118.33(11)$ & $\mathrm{N}(6)-\mathrm{C}(5)-\mathrm{C}(4)$ & $118.91(12)$ \\
$\mathrm{N}(6)-\mathrm{C}(5)-\mathrm{N}(7)$ & $118.44(13)$ & $\mathrm{H}(6 \mathrm{~A})-\mathrm{N}(6)-\mathrm{H}(6 \mathrm{~A}(6 \mathrm{~B})$ & 120.0 \\
$\mathrm{~N}(7)-\mathrm{C}(5)-\mathrm{C}(4)$ & $122.66(12)$ & $\mathrm{C}(5)-\mathrm{N}(7)-\mathrm{H}(7 \mathrm{~B})$ & 120.0 \\
$\mathrm{C}(5)-\mathrm{N}(6)-\mathrm{H}(6 \mathrm{~B})$ & 120.0 & $\mathrm{~N}(12)-\mathrm{C}(8)-\mathrm{N}(9)$ & $108.34(12)$ \\
$\mathrm{C}(5)-\mathrm{N}(7)-\mathrm{H}(7 \mathrm{~A})$ & 120.0 & $\mathrm{~N}(9)-\mathrm{C}(8)-\mathrm{C}(4)$ & $126.72(11)$ \\
$\mathrm{H}(7 \mathrm{~A})-\mathrm{N}(7)-\mathrm{H}(7 \mathrm{~B})$ & 120.0 & $\mathrm{C}(8)-\mathrm{N}(9)-\mathrm{H}(9)$ & 124.5 \\
$\mathrm{~N}(12)-\mathrm{C}(8)-\mathrm{C}(4)$ & $124.94(11)$ & $\mathrm{C}(11)-\mathrm{N}(10)-\mathrm{N}(9)$ & $102.03(11)$ \\
$\mathrm{C}(8)-\mathrm{N}(9)-\mathrm{N}(10)$ & $111.02(11)$ & $\mathrm{N}(10)-\mathrm{C}(11)-\mathrm{H}(11)$ & 122.3 \\
$\mathrm{~N}(10)-\mathrm{N}(9)-\mathrm{H}(9)$ & 124.5 & $\mathrm{C}(8)-\mathrm{N}(12)-\mathrm{C}(11)$ & $103.27(11)$ \\
$\mathrm{N}(10)-\mathrm{C}(11)-\mathrm{N}(12)$ & $115.34(13)$ & \multicolumn{2}{l}{} \\
$\mathrm{N}(12)-\mathrm{C}(11)-\mathrm{H}(11)$ & 122.3 & \multicolumn{2}{c}{}
\end{tabular}


Table S7. Torsion angles $\left[{ }^{\circ}\right]$ for 4 .

\begin{tabular}{lc}
\hline $\mathrm{O}(2)-\mathrm{N}(3)-\mathrm{C}(4)-\mathrm{C}(5)$ & $-178.72(12)$ \\
$\mathrm{O}(1)-\mathrm{N}(3)-\mathrm{C}(4)-\mathrm{C}(5)$ & $1.9(2)$ \\
$\mathrm{O}(2)-\mathrm{N}(3)-\mathrm{C}(4)-\mathrm{C}(8)$ & $0.6(2)$ \\
$\mathrm{O}(1)-\mathrm{N}(3)-\mathrm{C}(4)-\mathrm{C}(8)$ & $-178.87(12)$ \\
$\mathrm{N}(3)-\mathrm{C}(4)-\mathrm{C}(5)-\mathrm{N}(6)$ & $178.47(13)$ \\
$\mathrm{C}(8)-\mathrm{C}(4)-\mathrm{C}(5)-\mathrm{N}(6)$ & $-0.8(2)$ \\
$\mathrm{N}(3)-\mathrm{C}(4)-\mathrm{C}(5)-\mathrm{N}(7)$ & $-2.0(2)$ \\
$\mathrm{C}(8)-\mathrm{C}(4)-\mathrm{C}(5)-\mathrm{N}(7)$ & $178.76(13)$ \\
$\mathrm{N}(3)-\mathrm{C}(4)-\mathrm{C}(8)-\mathrm{N}(12)$ & $-179.96(13)$ \\
$\mathrm{C}(5)-\mathrm{C}(4)-\mathrm{C}(8)-\mathrm{N}(12)$ & $-0.7(2)$ \\
$\mathrm{N}(3)-\mathrm{C}(4)-\mathrm{C}(8)-\mathrm{N}(9)$ & $0.8(2)$ \\
$\mathrm{C}(5)-\mathrm{C}(4)-\mathrm{C}(8)-\mathrm{N}(9)$ & $-179.89(13)$ \\
$\mathrm{N}(12)-\mathrm{C}(8)-\mathrm{N}(9)-\mathrm{N}(10)$ & $-0.35(18)$ \\
$\mathrm{C}(4)-\mathrm{C}(8)-\mathrm{N}(9)-\mathrm{N}(10)$ & $178.96(13)$ \\
$\mathrm{C}(8)-\mathrm{N}(9)-\mathrm{N}(10)-\mathrm{C}(11)$ & $0.16(16)$ \\
$\mathrm{N}(9)-\mathrm{N}(10)-\mathrm{C}(11)-\mathrm{N}(12)$ & $0.09(16)$ \\
$\mathrm{N}(9)-\mathrm{C}(8)-\mathrm{N}(12)-\mathrm{C}(11)$ & $0.37(17)$ \\
$\mathrm{C}(4)-\mathrm{C}(8)-\mathrm{N}(12)-\mathrm{C}(11)$ & $-178.96(14)$ \\
$\mathrm{N}(10)-\mathrm{C}(11)-\mathrm{N}(12)-\mathrm{C}(8)$ & $-0.29(17)$ \\
\hline
\end{tabular}


Table S8. Hydrogen bonds for 4 [^ and ${ }^{\circ}$ ].

\begin{tabular}{lcccc}
\hline \multicolumn{1}{c}{ D-H...A } & $\mathrm{d}(\mathrm{D}-\mathrm{H})$ & $\mathrm{d}(\mathrm{H} . . . \mathrm{A})$ & $\mathrm{d}(\mathrm{D} \ldots \mathrm{A})$ & $<(\mathrm{DHA})$ \\
\hline $\mathrm{N}(6)-\mathrm{H}(6 \mathrm{~A}) \ldots \mathrm{O}(1) \# 1$ & 0.86 & 2.18 & $2.9851(16)$ & 156.5 \\
$\mathrm{~N}(6)-\mathrm{H}(6 \mathrm{~B}) \ldots \mathrm{N}(12)$ & 0.86 & 1.96 & $2.6619(17)$ & 138.4 \\
$\mathrm{~N}(7)-\mathrm{H}(7 \mathrm{~A}) \ldots \mathrm{O}(1) \# 1$ & 0.86 & 2.40 & $3.1506(17)$ & 146.6 \\
$\mathrm{~N}(7)-\mathrm{H}(7 \mathrm{~A}) \ldots \mathrm{O}(2) \# 1$ & 0.86 & 2.36 & $3.0866(16)$ & 141.8 \\
$\mathrm{~N}(7)-\mathrm{H}(7 \mathrm{~B}) \ldots \mathrm{O}(1)$ & 0.86 & 1.92 & $2.5633(17)$ & 130.2 \\
$\mathrm{~N}(7)-\mathrm{H}(7 \mathrm{~B}) \ldots \mathrm{N}(10) \# 2$ & 0.86 & 2.60 & $3.1400(18)$ & 121.6 \\
$\mathrm{~N}(9)-\mathrm{H}(9) \ldots \mathrm{O}(2)$ & 0.86 & 2.01 & $2.5509(16)$ & 119.9 \\
$\mathrm{C}(11)-\mathrm{H}(11) \ldots \mathrm{O}(2) \# 3$ & 0.93 & 2.51 & $3.2449(19)$ & 135.8
\end{tabular}

Symmetry transformations used to generate equivalent atoms:

$\# 1 \mathrm{x}+1 / 2, \mathrm{y},-\mathrm{z}+3 / 2 \quad \# 2-\mathrm{x}+1 / 2,-\mathrm{y}+1, \mathrm{z}+1 / 2 \quad \# 3 \mathrm{x}+1 / 2,-\mathrm{y}+1 / 2,-\mathrm{z}+1$ 
Table S9. Crystal data and structure refinement for $\mathbf{5}$.

\begin{tabular}{|c|c|c|}
\hline Identification code & \multicolumn{2}{|l|}{5} \\
\hline CCDC number & \multicolumn{2}{|l|}{1975692} \\
\hline Empirical formula & \multicolumn{2}{|l|}{$\mathrm{C}_{5} \mathrm{H}_{7} \mathrm{~N}_{7} \mathrm{O}_{2}$} \\
\hline Formula weight & \multicolumn{2}{|l|}{197.18} \\
\hline Temperature & \multicolumn{2}{|l|}{$296(2) \mathrm{K}$} \\
\hline Wavelength & \multicolumn{2}{|l|}{$0.71073 \AA$} \\
\hline Crystal system & \multicolumn{2}{|l|}{ Monoclinic } \\
\hline Space group & \multicolumn{2}{|l|}{$\mathrm{P} 2 / \mathrm{n}$} \\
\hline Unit cell dimensions & $\begin{array}{l}a=8.0844(9) \AA \\
b=9.0540(11) \AA \\
c=10.5384(12) \AA\end{array}$ & $\begin{array}{l}\alpha=90^{\circ} . \\
\beta=96.114(3)^{\circ} . \\
\gamma=90^{\circ} .\end{array}$ \\
\hline Volume & \multicolumn{2}{|l|}{$766.98(15) \AA^{3}$} \\
\hline $\mathrm{Z}$ & \multicolumn{2}{|l|}{4} \\
\hline Density $\left(20^{\circ} \mathrm{C}\right)$ & \multicolumn{2}{|l|}{$1.708 \mathrm{Mg} / \mathrm{m}^{3}$} \\
\hline $\begin{array}{l}\text { Absorption coefficient } \\
F(000)\end{array}$ & \multicolumn{2}{|l|}{$0.138 \mathrm{~mm}^{-1}$} \\
\hline Crystal size & \multicolumn{2}{|c|}{$0.205 \times 0.107 \times 0.030 \mathrm{~mm}^{3}$} \\
\hline Theta range for data collection & \multicolumn{2}{|l|}{2.249 to $29.983^{\circ}$} \\
\hline Index ranges & \multicolumn{2}{|c|}{$-11<=\mathrm{h}<=11,-11<=\mathrm{k}<=12,-14<=1<=14$} \\
\hline Reflections collected & \multicolumn{2}{|l|}{8612} \\
\hline Independent reflections & \multicolumn{2}{|l|}{$2188\left[\mathrm{R}_{\mathrm{int}}=0.0645\right]$} \\
\hline Completeness to theta $=25.242^{\circ}$ & \multicolumn{2}{|l|}{$99.9 \%$} \\
\hline Absorption correction & \multicolumn{2}{|c|}{ Semi-empirical from equivalents } \\
\hline Max. and min. transmission & \multicolumn{2}{|l|}{0.7460 and 0.6681} \\
\hline Refinement method & \multirow{2}{*}{\multicolumn{2}{|c|}{$\begin{array}{l}\text { Full-matrix least-squares on } \mathrm{F}^{2} \\
2188 \text { / } 0 \text { / } 127\end{array}$}} \\
\hline Data / restraints / parameters & & \\
\hline Goodness-of-fit on $\mathrm{F}^{2}$ & \multicolumn{2}{|l|}{1.015} \\
\hline Final $R$ indices $[\mathrm{I}>2 \operatorname{sigma}(\mathrm{I})]$ & \multicolumn{2}{|c|}{$\mathrm{R}_{1}=0.0554, \mathrm{wR}_{2}=0.1140$} \\
\hline $\mathrm{R}$ indices (all data) & \multicolumn{2}{|c|}{$\mathrm{R}_{1}=0.1335, \mathrm{wR}_{2}=0.1382$} \\
\hline Largest diff. peak and hole & \multicolumn{2}{|c|}{0.268 and -0.231 e. $\AA^{-3}$} \\
\hline
\end{tabular}


Table S10. Bond lengths $\left[\AA ̊ \AA\right.$ and angles $\left[{ }^{\circ}\right]$ for 5.

\begin{tabular}{|c|c|c|c|}
\hline $\mathrm{O}(6)-\mathrm{N}(5)$ & $1.255(2)$ & $\mathrm{O}(7)-\mathrm{N}(5)$ & $1.272(2)$ \\
\hline $\mathrm{N}(14)-\mathrm{C}(8)$ & $1.334(3)$ & $\mathrm{N}(14)-\mathrm{C}(12)$ & $1.351(3)$ \\
\hline $\mathrm{N}(5)-\mathrm{C}(4)$ & $1.375(3)$ & $\mathrm{N}(9)-\mathrm{C}(10)$ & $1.332(3)$ \\
\hline $\mathrm{N}(9)-\mathrm{C}(8)$ & $1.352(3)$ & $\mathrm{N}(13)-\mathrm{C}(12)$ & $1.326(3)$ \\
\hline $\mathrm{N}(13)-\mathrm{H}(13 \mathrm{~A})$ & 0.8600 & $\mathrm{~N}(13)-\mathrm{H}(13 \mathrm{~B})$ & 0.8600 \\
\hline $\mathrm{N}(11)-\mathrm{C}(10)$ & $1.313(3)$ & $\mathrm{N}(11)-\mathrm{C}(12)$ & $1.348(3)$ \\
\hline $\mathrm{N}(1)-\mathrm{C}(3)$ & $1.310(3)$ & $\mathrm{N}(1)-\mathrm{H}(1 \mathrm{~A})$ & 0.8600 \\
\hline $\mathrm{N}(1)-\mathrm{H}(1 \mathrm{~B})$ & 0.8600 & $\mathrm{~N}(2)-\mathrm{C}(3)$ & $1.316(3)$ \\
\hline $\mathrm{N}(2)-\mathrm{H}(2 \mathrm{~A})$ & 0.8600 & $\mathrm{~N}(2)-\mathrm{H}(2 \mathrm{~B})$ & 0.8600 \\
\hline $\mathrm{C}(8)-\mathrm{C}(4)$ & $1.477(3)$ & $\mathrm{C}(3)-\mathrm{C}(4)$ & $1.456(3)$ \\
\hline $\mathrm{C}(10)-\mathrm{H}(10)$ & 0.9300 & & \\
\hline$C(8)-N(14)-C(12)$ & $115.4(2)$ & $\mathrm{O}(6)-\mathrm{N}(5)-\mathrm{O}(7) 117.98(18)$ & \\
\hline $\mathrm{O}(6)-\mathrm{N}(5)-\mathrm{C}(4)$ & $121.22(18)$ & $\mathrm{O}(7)-\mathrm{N}(5)-\mathrm{C}(4) 120.76(18)$ & \\
\hline $\mathrm{C}(10)-\mathrm{N}(9)-\mathrm{C}(8)$ & $114.9(2)$ & $\mathrm{C}(12)-\mathrm{N}(13)-\mathrm{H}(13 \mathrm{~A})$ & 120.0 \\
\hline $\mathrm{C}(12)-\mathrm{N}(13)-\mathrm{H}(13 \mathrm{~B})$ & 120.0 & $\mathrm{H}(13 \mathrm{~A})-\mathrm{N}(13)-\mathrm{H}(13 \mathrm{~B})$ & 120.0 \\
\hline $\mathrm{C}(10)-\mathrm{N}(11)-\mathrm{C}(12)$ & $113.9(2)$ & $\mathrm{C}(3)-\mathrm{N}(1)-\mathrm{H}(1 \mathrm{~A})$ & 120.0 \\
\hline $\mathrm{C}(3)-\mathrm{N}(1)-\mathrm{H}(1 \mathrm{~B})$ & 120.0 & $\mathrm{H}(1 \mathrm{~A})-\mathrm{N}(1)-\mathrm{H}(1 \mathrm{~B})$ & 120.0 \\
\hline $\mathrm{C}(3)-\mathrm{N}(2)-\mathrm{H}(2 \mathrm{~A})$ & 120.0 & $\mathrm{C}(3)-\mathrm{N}(2)-\mathrm{H}(2 \mathrm{~B})$ & 120.0 \\
\hline $\mathrm{H}(2 \mathrm{~A})-\mathrm{N}(2)-\mathrm{H}(2 \mathrm{~B})$ & 120.0 & $\mathrm{~N}(14)-\mathrm{C}(8)-\mathrm{N}(9)$ & $123.7(2)$ \\
\hline $\mathrm{N}(14)-\mathrm{C}(8)-\mathrm{C}(4)$ & $120.3(2)$ & $\mathrm{N}(9)-\mathrm{C}(8)-\mathrm{C}(4)$ & $116.0(2)$ \\
\hline $\mathrm{N}(1)-\mathrm{C}(3)-\mathrm{N}(2)$ & 115.9(2) & $\mathrm{N}(1)-\mathrm{C}(3)-\mathrm{C}(4)$ & $124.6(2)$ \\
\hline $\mathrm{N}(2)-\mathrm{C}(3)-\mathrm{C}(4)$ & $119.5(2)$ & $\mathrm{N}(5)-\mathrm{C}(4)-\mathrm{C}(3) 119.78(19)$ & \\
\hline $\mathrm{N}(5)-\mathrm{C}(4)-\mathrm{C}(8)$ & $118.63(19)$ & $\mathrm{C}(3)-\mathrm{C}(4)-\mathrm{C}(8) 121.59(19)$ & \\
\hline $\mathrm{N}(13)-\mathrm{C}(12)-\mathrm{N}(11)$ & $115.6(2)$ & $\mathrm{N}(13)-\mathrm{C}(12)-\mathrm{N}(14)$ & $119.4(2)$ \\
\hline $\mathrm{N}(11)-\mathrm{C}(12)-\mathrm{N}(14)$ & $125.0(2)$ & $\mathrm{N}(11)-\mathrm{C}(10)-\mathrm{N}(9)$ & $127.0(2)$ \\
\hline $\mathrm{N}(11)-\mathrm{C}(10)-\mathrm{H}(10)$ & 116.5 & $\mathrm{~N}(9)-\mathrm{C}(10)-\mathrm{H}(10)$ & 116.5 \\
\hline
\end{tabular}


Table S11. Torsion angles $\left[{ }^{\circ}\right]$ for 5 .

\begin{tabular}{lc}
\hline $\mathrm{C}(12)-\mathrm{N}(14)-\mathrm{C}(8)-\mathrm{N}(9)$ & $2.0(3)$ \\
$\mathrm{C}(12)-\mathrm{N}(14)-\mathrm{C}(8)-\mathrm{C}(4)$ & $179.2(2)$ \\
$\mathrm{C}(10)-\mathrm{N}(9)-\mathrm{C}(8)-\mathrm{N}(14)$ & $-1.2(3)$ \\
$\mathrm{C}(10)-\mathrm{N}(9)-\mathrm{C}(8)-\mathrm{C}(4)$ & $-178.4(2)$ \\
$\mathrm{O}(6)-\mathrm{N}(5)-\mathrm{C}(4)-\mathrm{C}(3)$ & $-168.7(2)$ \\
$\mathrm{O}(7)-\mathrm{N}(5)-\mathrm{C}(4)-\mathrm{C}(3)$ & $8.9(3)$ \\
$\mathrm{O}(6)-\mathrm{N}(5)-\mathrm{C}(4)-\mathrm{C}(8)$ & $10.9(3)$ \\
$\mathrm{O}(7)-\mathrm{N}(5)-\mathrm{C}(4)-\mathrm{C}(8)$ & $-171.5(2)$ \\
$\mathrm{N}(1)-\mathrm{C}(3)-\mathrm{C}(4)-\mathrm{N}(5)$ & $-3.0(4)$ \\
$\mathrm{N}(2)-\mathrm{C}(3)-\mathrm{C}(4)-\mathrm{N}(5)$ & $177.4(2)$ \\
$\mathrm{N}(1)-\mathrm{C}(3)-\mathrm{C}(4)-\mathrm{C}(8)$ & $177.4(2)$ \\
$\mathrm{N}(2)-\mathrm{C}(3)-\mathrm{C}(4)-\mathrm{C}(8)$ & $-2.2(4)$ \\
$\mathrm{N}(14)-\mathrm{C}(8)-\mathrm{C}(4)-\mathrm{N}(5)$ & $33.8(3)$ \\
$\mathrm{N}(9)-\mathrm{C}(8)-\mathrm{C}(4)-\mathrm{N}(5)$ & $-148.8(2)$ \\
$\mathrm{N}(14)-\mathrm{C}(8)-\mathrm{C}(4)-\mathrm{C}(3)$ & $-146.6(2)$ \\
$\mathrm{N}(9)-\mathrm{C}(8)-\mathrm{C}(4)-\mathrm{C}(3)$ & $30.8(3)$ \\
$\mathrm{C}(10)-\mathrm{N}(11)-\mathrm{C}(12)-\mathrm{N}(13)$ & $178.2(2)$ \\
$\mathrm{C}(10)-\mathrm{N}(11)-\mathrm{C}(12)-\mathrm{N}(14)$ & $-0.6(3)$ \\
$\mathrm{C}(8)-\mathrm{N}(14)-\mathrm{C}(12)-\mathrm{N}(13)$ & $-179.9(2)$ \\
$\mathrm{C}(8)-\mathrm{N}(14)-\mathrm{C}(12)-\mathrm{N}(11)$ & $-1.1(3)$ \\
$\mathrm{C}(12)-\mathrm{N}(11)-\mathrm{C}(10)-\mathrm{N}(9)$ & $1.6(4)$ \\
$\mathrm{C}(8)-\mathrm{N}(9)-\mathrm{C}(10)-\mathrm{N}(11)$ & $-0.8(4)$ \\
\hline
\end{tabular}

Table S12. Hydrogen bonds for 5 [^ and $\left.{ }^{\circ}\right]$.

\begin{tabular}{lcccc}
\hline \multicolumn{1}{c}{ D-H...A } & $\mathrm{d}(\mathrm{D}-\mathrm{H})$ & $\mathrm{d}(\mathrm{H} \ldots \mathrm{A})$ & $\mathrm{d}(\mathrm{D} \ldots \mathrm{A})$ & $<(\mathrm{DHA})$ \\
\hline $\mathrm{N}(13)-\mathrm{H}(13 \mathrm{~B}) \ldots \mathrm{N}(14) \# 2$ & 0.86 & 2.17 & $3.018(3)$ & 167.5 \\
$\mathrm{~N}(1)-\mathrm{H}(1 \mathrm{~A}) \ldots \mathrm{O}(6) \# 3$ & 0.86 & 2.23 & $3.083(3)$ & 175.7 \\
$\mathrm{~N}(1)-\mathrm{H}(1 \mathrm{~B}) \ldots \mathrm{O}(7)$ & 0.86 & 1.95 & $2.577(3)$ & 128.5 \\
$\mathrm{~N}(2)-\mathrm{H}(2 \mathrm{~A}) \ldots \mathrm{O}(7) \# 3$ & 0.86 & 2.09 & $2.939(3)$ & 169.8 \\
$\mathrm{~N}(2)-\mathrm{H}(2 \mathrm{~B}) \ldots \mathrm{N}(9)$ & 0.86 & 1.98 & $2.640(3)$ & 132.1 \\
& & & & \\
\hline
\end{tabular}

Symmetry transformations used to generate equivalent atoms:

\#1 $\mathrm{x}, \mathrm{y}+1, \mathrm{z} \quad \# 2-\mathrm{x},-\mathrm{y}+1,-\mathrm{z}+1 \quad \# 3 \mathrm{x}+1 / 2,-\mathrm{y}, \mathrm{z}+1 / 2$

\#4 x,y-1,z 
Table S13. Crystal data and structure refinement for 9.

\begin{tabular}{|c|c|c|}
\hline Identification code & \multicolumn{2}{|l|}{9} \\
\hline CCDC number & \multicolumn{2}{|l|}{1975693} \\
\hline Empirical formula & \multicolumn{2}{|l|}{$\mathrm{C}_{3} \mathrm{H}_{8} \mathrm{~N}_{8} \mathrm{O}_{2}$} \\
\hline Formula weight & \multicolumn{2}{|l|}{188.17} \\
\hline Temperature & \multicolumn{2}{|l|}{ 293(2) K } \\
\hline Wavelength & \multicolumn{2}{|l|}{$0.71073 \AA$} \\
\hline Crystal system & \multicolumn{2}{|l|}{ Orthorhombic } \\
\hline Space group & \multicolumn{2}{|l|}{ Pna2 ${ }_{1}$} \\
\hline Unit cell dimensions & $\begin{array}{l}a=8.4672(4) \AA \\
b=11.1265(6) \AA \\
c=7.9409(4) \AA\end{array}$ & $\begin{array}{l}\alpha=90^{\circ} . \\
\beta=90^{\circ} . \\
\gamma=90^{\circ} .\end{array}$ \\
\hline Volume & \multicolumn{2}{|l|}{$748.11(7) \AA^{3}$} \\
\hline $\mathrm{Z}$ & \multicolumn{2}{|l|}{4} \\
\hline Density $\left(20^{\circ} \mathrm{C}\right)$ & \multicolumn{2}{|l|}{$1.671 \mathrm{Mg} / \mathrm{m}^{3}$} \\
\hline $\begin{array}{l}\text { Absorption coefficient } \\
F(000)\end{array}$ & \multicolumn{2}{|l|}{$0.140 \mathrm{~mm}^{-1}$} \\
\hline Crystal size & \multicolumn{2}{|c|}{$0.418 \times 0.093 \times 0.028 \mathrm{~mm}^{3}$} \\
\hline Theta range for data collection & \multicolumn{2}{|c|}{3.023 to $30.042^{\circ}$. } \\
\hline Index ranges & \multicolumn{2}{|c|}{$-11<=\mathrm{h}<=11,-15<=\mathrm{k}<=15,-10<=1<=10$} \\
\hline Reflections collected & \multicolumn{2}{|l|}{6901} \\
\hline Independent reflections & \multicolumn{2}{|c|}{$1991\left[\mathrm{R}_{\mathrm{int}}=0.0218\right]$} \\
\hline Completeness to theta $=25.242^{\circ}$ & \multicolumn{2}{|l|}{$100.0 \%$} \\
\hline Absorption correction & \multicolumn{2}{|c|}{ Semi-empirical from equivalents } \\
\hline Max. and min. transmission & \multicolumn{2}{|c|}{0.7460 and 0.6840} \\
\hline $\begin{array}{l}\text { Refinement method } \\
\text { Data / restraints / parameters }\end{array}$ & \multicolumn{2}{|c|}{$\begin{array}{l}\text { Full-matrix least-squares on } \mathrm{F}^{2} \\
1991 / 9 / 130\end{array}$} \\
\hline Goodness-of-fit on $\mathrm{F}^{2}$ & \multicolumn{2}{|l|}{1.062} \\
\hline Final $R$ indices $[I>2 \operatorname{sigma}(I)]$ & \multicolumn{2}{|c|}{$\mathrm{R}_{1}=0.0333, \mathrm{wR}_{2}=0.0831$} \\
\hline $\mathrm{R}$ indices (all data) & \multicolumn{2}{|c|}{$\mathrm{R}_{1}=0.0402, \mathrm{wR}_{2}=0.0870$} \\
\hline Absolute structure parameter & \multicolumn{2}{|c|}{$-0.5(7)$} \\
\hline Largest diff. peak and hole & \multicolumn{2}{|c|}{0.289 and -0.205 e. $\AA^{-}-3$} \\
\hline
\end{tabular}


Table S14. Bond lengths $[\AA]$ and angles $\left[{ }^{\circ}\right]$ for 9.

\begin{tabular}{llll}
\hline $\mathrm{O}(1)-\mathrm{N}(3)$ & $1.277(2)$ & $\mathrm{O}(2)-\mathrm{N}(3)$ & $1.271(2)$ \\
$\mathrm{N}(3)-\mathrm{C}(4)$ & $1.348(3)$ & $\mathrm{C}(4)-\mathrm{C}(5)$ & $1.443(3)$ \\
$\mathrm{C}(4)-\mathrm{C}(8)$ & $1.471(2)$ & $\mathrm{C}(5)-\mathrm{N}(7)$ & $1.316(3)$ \\
$\mathrm{C}(5)-\mathrm{N}(6)$ & $1.326(2)$ & $\mathrm{N}(6)-\mathrm{H}(6 \mathrm{~A})$ & 0.8600 \\
$\mathrm{~N}(6)-\mathrm{H}(6 \mathrm{~B})$ & 0.8600 & $\mathrm{~N}(7)-\mathrm{H}(7 \mathrm{~A})$ & 0.8600 \\
$\mathrm{~N}(7)-\mathrm{H}(7 \mathrm{~B})$ & 0.8600 & $\mathrm{C}(8)-\mathrm{N}(12)$ & $1.335(3)$ \\
$\mathrm{C}(8)-\mathrm{N}(9)$ & $1.343(3)$ & $\mathrm{N}(1)-\mathrm{N}(10)-\mathrm{N}(12)$ & $1.346(2)$ \\
$\mathrm{N}(10)-\mathrm{N}(11)$ & $1.315(3)$ & $\mathrm{N}(13)-\mathrm{H}(13 \mathrm{~B})$ & $1.336(2)$ \\
$\mathrm{N}(13)-\mathrm{H}(13 \mathrm{~A})$ & $0.907(11)$ & $\mathrm{N}(13)-\mathrm{H}(13 \mathrm{D})$ & $0.911(12)$ \\
$\mathrm{N}(13)-\mathrm{H}(13 \mathrm{C})$ & $0.908(12)$ & & $0.914(12)$ \\
& & $\mathrm{O}(2)-\mathrm{N}(3)-\mathrm{C}(4)$ & \\
$\mathrm{O}(2)-\mathrm{N}(3)-\mathrm{O}(1)$ & $117.66(17)$ & $\mathrm{C}(3)-\mathrm{C}(4)-\mathrm{C}(5)$ & $123.05(18)-\mathrm{C}(8)$ \\
$\mathrm{O}(1)-\mathrm{N}(3)-\mathrm{C}(4)$ & $119.29(15)$ & $\mathrm{N}(7)-\mathrm{C}(5)-\mathrm{C}(4)$ & $120.53(15)$ \\
$\mathrm{N}(3)-\mathrm{C}(4)-\mathrm{C}(8)$ & $118.48(18)$ & $\mathrm{C}(5)-\mathrm{N}(6)-\mathrm{H}(6 \mathrm{~A})$ & $118.08(17)$ \\
$\mathrm{N}(7)-\mathrm{C}(5)-\mathrm{N}(6)$ & $118.97(19)$ & $\mathrm{H}(6 \mathrm{~A})-\mathrm{N}(6)-\mathrm{H}(6 \mathrm{~B})$ & 120.0 \\
$\mathrm{~N}(6)-\mathrm{C}(5)-\mathrm{C}(4)$ & $122.94(19)$ & $\mathrm{C}(5)-\mathrm{N}(7)-\mathrm{H}(7 \mathrm{~B})$ & 120.0 \\
$\mathrm{C}(5)-\mathrm{N}(6)-\mathrm{H}(6 \mathrm{~B})$ & 120.0 & $\mathrm{~N}(12)-\mathrm{C}(8)-\mathrm{N}(9)$ & $111.10(14)$ \\
$\mathrm{C}(5)-\mathrm{N}(7)-\mathrm{H}(7 \mathrm{~A})$ & 120.0 & $\mathrm{~N}(9)-\mathrm{C}(8)-\mathrm{C}(4)$ & $123.33(19)$ \\
$\mathrm{H}(7 \mathrm{~A})-\mathrm{N}(7)-\mathrm{H}(7 \mathrm{~B})$ & 120.0 & $\mathrm{~N}(11)-\mathrm{N}(10)-\mathrm{N}(9)$ & $109.13(18)$ \\
$\mathrm{N}(12)-\mathrm{C}(8)-\mathrm{C}(4)$ & $125.6(2)$ & $\mathrm{C}(8)-\mathrm{N}(12)-\mathrm{N}(11)$ & $105.17(18)$ \\
$\mathrm{C}(8)-\mathrm{N}(9)-\mathrm{N}(10)$ & $104.82(17)$ & $\mathrm{H}(13 \mathrm{~A})-\mathrm{N}(13)-\mathrm{H}(13 \mathrm{C})$ & $109(2)$ \\
$\mathrm{N}(10)-\mathrm{N}(11)-\mathrm{N}(12)$ & $109.77(18)$ & $\mathrm{H}(13 \mathrm{~A})-\mathrm{N}(13)-\mathrm{H}(13 \mathrm{D})$ & $111(2)$ \\
$\mathrm{H}(13 \mathrm{~A})-\mathrm{N}(13)-\mathrm{H}(13 \mathrm{~B})$ & $111(2)$ & $\mathrm{H}(13 \mathrm{C})-\mathrm{N}(13)-\mathrm{H}(13 \mathrm{D})$ & $112(2)$ \\
$\mathrm{H}(13 \mathrm{~B})-\mathrm{N}(13)-\mathrm{H}(13 \mathrm{C})$ & $104(3)$ & & \\
$\mathrm{H}(13 \mathrm{~B})-\mathrm{N}(13)-\mathrm{H}(13 \mathrm{D})$ & $110(2)$ & & \\
\hline & & &
\end{tabular}

Table S15. Torsion angles $\left[{ }^{\circ}\right]$ for 9.

\begin{tabular}{lc}
\hline $\mathrm{O}(2)-\mathrm{N}(3)-\mathrm{C}(4)-\mathrm{C}(5)$ & $-4.1(4)$ \\
$\mathrm{O}(1)-\mathrm{N}(3)-\mathrm{C}(4)-\mathrm{C}(5)$ & $175.1(2)$ \\
$\mathrm{O}(2)-\mathrm{N}(3)-\mathrm{C}(4)-\mathrm{C}(8)$ & $177.8(2)$ \\
$\mathrm{O}(1)-\mathrm{N}(3)-\mathrm{C}(4)-\mathrm{C}(8)$ & $-3.0(3)$ \\
$\mathrm{N}(3)-\mathrm{C}(4)-\mathrm{C}(5)-\mathrm{N}(7)$ & $-174.6(2)$ \\
$\mathrm{C}(8)-\mathrm{C}(4)-\mathrm{C}(5)-\mathrm{N}(7)$ & $3.4(3)$ \\
$\mathrm{N}(3)-\mathrm{C}(4)-\mathrm{C}(5)-\mathrm{N}(6)$ & $5.5(4)$ \\
$\mathrm{C}(8)-\mathrm{C}(4)-\mathrm{C}(5)-\mathrm{N}(6)$ & $-176.5(2)$ \\
$\mathrm{N}(3)-\mathrm{C}(4)-\mathrm{C}(8)-\mathrm{N}(12)$ & $66.0(3)$ \\
$\mathrm{C}(5)-\mathrm{C}(4)-\mathrm{C}(8)-\mathrm{N}(12)$ & $-112.1(3)$ \\
$\mathrm{N}(3)-\mathrm{C}(4)-\mathrm{C}(8)-\mathrm{N}(9)$ & $-114.7(3)$ \\
$\mathrm{C}(5)-\mathrm{C}(4)-\mathrm{C}(8)-\mathrm{N}(9)$ & $67.2(3)$ \\
$\mathrm{N}(12)-\mathrm{C}(8)-\mathrm{N}(9)-\mathrm{N}(10)$ & $-0.4(3)$ \\
$\mathrm{C}(4)-\mathrm{C}(8)-\mathrm{N}(9)-\mathrm{N}(10)$ & $-179.8(2)$ \\
$\mathrm{C}(8)-\mathrm{N}(9)-\mathrm{N}(10)-\mathrm{N}(11)$ & $0.1(2)$ \\
$\mathrm{N}(9)-\mathrm{N}(10)-\mathrm{N}(11)-\mathrm{N}(12)$ & $0.3(2)$ \\
$\mathrm{N}(9)-\mathrm{C}(8)-\mathrm{N}(12)-\mathrm{N}(11)$ & $0.6(3)$ \\
$\mathrm{C}(4)-\mathrm{C}(8)-\mathrm{N}(12)-\mathrm{N}(11)$ & $180.0(2)$ \\
$\mathrm{N}(10)-\mathrm{N}(11)-\mathrm{N}(12)-\mathrm{C}(8)$ & $-0.6(2)$ \\
\hline
\end{tabular}


Table S16. Hydrogen bonds for 9 [A and $\left.{ }^{\circ}\right]$.

\begin{tabular}{lcccc}
\hline \multicolumn{1}{c}{ D-H...A } & $\mathrm{d}(\mathrm{D}-\mathrm{H})$ & $\mathrm{d}(\mathrm{H} \ldots \mathrm{A})$ & $\mathrm{d}(\mathrm{D} \ldots \mathrm{A})$ & $<(\mathrm{DHA})$ \\
\hline $\mathrm{N}(7)-\mathrm{H}(7 \mathrm{~A}) \ldots \mathrm{O}(1) \# 1$ & 0.86 & 2.00 & $2.848(2)$ & 166.9 \\
$\mathrm{~N}(7)-\mathrm{H}(7 \mathrm{~B}) \ldots \mathrm{O}(1) \# 2$ & 0.86 & 2.05 & $2.779(2)$ & 141.3 \\
$\mathrm{~N}(6)-\mathrm{H}(6 \mathrm{~B}) \ldots \mathrm{O}(2)$ & 0.86 & 1.96 & $2.590(2)$ & 129.2 \\
$\mathrm{~N}(13)-\mathrm{H}(13 \mathrm{~A}) \ldots \mathrm{N}(9) \# 4$ & $0.907(11)$ & $2.057(15)$ & $2.924(3)$ & $159(2)$ \\
$\mathrm{N}(13)-\mathrm{H}(13 \mathrm{~B}) \ldots \mathrm{N}(11) \# 5$ & $0.911(12)$ & $2.162(13)$ & $3.072(3)$ & $176(3)$ \\
$\mathrm{N}(13)-\mathrm{H}(13 \mathrm{C}) \ldots \mathrm{N}(12) \# 6$ & $0.908(12)$ & $2.14(2)$ & $2.930(3)$ & $145(2)$ \\
$\mathrm{N}(13)-\mathrm{H}(13 \mathrm{D}) \ldots \mathrm{N}(10) \# 3$ & $0.914(12)$ & $2.178(13)$ & $3.090(3)$ & $175(3)$ \\
& & & &
\end{tabular}

Symmetry transformations used to generate equivalent atoms:

$\# 1-\mathrm{x}+3 / 2, \mathrm{y}+1 / 2, \mathrm{z}+1 / 2 \quad \# 2-\mathrm{x}+1,-\mathrm{y}+1, \mathrm{z}+1 / 2 \quad \# 3 \mathrm{x}+1, \mathrm{y}, \mathrm{z}$

$\# 4 \mathrm{x}+1 / 2,-\mathrm{y}+3 / 2, \mathrm{z} \quad \# 5-\mathrm{x}+1,-\mathrm{y}+1, \mathrm{z}-1 / 2 \quad \# 6-\mathrm{x}+3 / 2, \mathrm{y}+1 / 2, \mathrm{z}-1 / 2$

Table S17. Crystal data and structure refinement for $\mathbf{1 0}$.

\begin{tabular}{|c|c|c|}
\hline Identification code & \multicolumn{2}{|l|}{$\mathbf{1 0}$} \\
\hline CCDC number & \multicolumn{2}{|l|}{1975700} \\
\hline Empirical formula & \multicolumn{2}{|l|}{$\mathrm{C}_{3} \mathrm{H}_{4} \mathrm{~N}_{8} \mathrm{O}_{4}$} \\
\hline Formula weight & \multicolumn{2}{|l|}{216.14} \\
\hline Temperature & \multicolumn{2}{|l|}{$293(2) \mathrm{K}$} \\
\hline Wavelength & \multicolumn{2}{|l|}{$1.54178 \AA$} \\
\hline Crystal system & \multicolumn{2}{|l|}{ Triclinic } \\
\hline Space group & \multicolumn{2}{|l|}{$\mathrm{P}-1$} \\
\hline Unit cell dimensions & $\begin{array}{l}a=7.3380(4) \AA \\
b=7.4214(4) \AA \\
c=8.9759(4) \AA\end{array}$ & $\begin{array}{l}\alpha=92.332(3)^{\circ} . \\
\beta=102.352(4)^{\circ} . \\
\gamma=118.864(3)^{\circ} .\end{array}$ \\
\hline Volume & \multicolumn{2}{|l|}{$412.21(4) \AA^{3}$} \\
\hline $\mathrm{Z}$ & \multicolumn{2}{|l|}{2} \\
\hline Density $\left(20^{\circ} \mathrm{C}\right)$ & \multicolumn{2}{|l|}{$1.741 \mathrm{Mg} / \mathrm{m}^{3}$} \\
\hline $\begin{array}{l}\text { Absorption coefficient } \\
F(000)\end{array}$ & \multicolumn{2}{|l|}{$1.394 \mathrm{~mm}^{-1}$} \\
\hline Crystal size & \multicolumn{2}{|c|}{$0.301 \times 0.120 \times 0.023 \mathrm{~mm}^{3}$} \\
\hline Theta range for data collection & \multicolumn{2}{|c|}{5.117 to $74.617^{\circ}$. } \\
\hline Index ranges & \multicolumn{2}{|c|}{$-7<=\mathrm{h}<=8,-8<=\mathrm{k}<=7,-10<=\mathrm{l}<=11$} \\
\hline Reflections collected & \multicolumn{2}{|c|}{2803} \\
\hline Independent reflections & \multicolumn{2}{|c|}{$1384\left[\mathrm{R}_{\mathrm{int}}=0.0366\right]$} \\
\hline Completeness to theta $=67.679^{\circ}$ & \multicolumn{2}{|c|}{$86.6 \%$} \\
\hline Absorption correction & \multicolumn{2}{|c|}{ Semi-empirical from equivalents } \\
\hline Max. and min. transmission & \multicolumn{2}{|c|}{0.7538 and 0.6041} \\
\hline Refinement method & \multicolumn{2}{|c|}{ Full-matrix least-squares on $\mathrm{F}^{2}$} \\
\hline Data / restraints / parameters & \multicolumn{2}{|l|}{1384 / 0 / 136} \\
\hline Goodness-of-fit on $\mathrm{F}^{2}$ & \multicolumn{2}{|l|}{1.087} \\
\hline Final $\mathrm{R}$ indices [I>2sigma(I)] & \multicolumn{2}{|c|}{$\mathrm{R}_{1}=0.0394, \mathrm{wR}_{2}=0.1099$} \\
\hline $\mathrm{R}$ indices (all data) & \multicolumn{2}{|c|}{$\mathrm{R}_{1}=0.0457, \mathrm{wR}_{2}=0.1137$} \\
\hline Largest diff. peak and hole & \multicolumn{2}{|c|}{0.349 and -0.219 e. $\AA^{-3}$} \\
\hline
\end{tabular}


Table S18. Bond lengths $[\AA]$ and angles $\left[^{\circ}\right]$ for $\mathbf{1 0}$.

\begin{tabular}{lllr}
\hline $\mathrm{N}(1)-\mathrm{C}(5)$ & $1.326(2)$ & $\mathrm{N}(1)-\mathrm{N}(2)$ & $1.335(2)$ \\
$\mathrm{N}(2)-\mathrm{N}(3)$ & $1.310(2)$ & $\mathrm{N}(3)-\mathrm{N}(4)$ & $1.332(2)$ \\
$\mathrm{N}(4)-\mathrm{C}(5)$ & $1.324(3)$ & $\mathrm{C}(5)-\mathrm{C}(6)$ & $1.498(2)$ \\
$\mathrm{C}(6)-\mathrm{N}(7)$ & $1.535(2)$ & $\mathrm{C}(6)-\mathrm{C}(13)$ & $1.540(2)$ \\
$\mathrm{C}(6)-\mathrm{N}(10)$ & $1.577(2)$ & $\mathrm{N}(7)-\mathrm{O}(8)$ & $1.190(2)$ \\
$\mathrm{N}(7)-\mathrm{O}(9)$ & $1.208(2)$ & $\mathrm{N}(10)-\mathrm{O}(12)$ & $1.189(2)$ \\
$\mathrm{N}(10)-\mathrm{O}(11)$ & $1.201(2)$ & $\mathrm{N}(13)-\mathrm{N}(14)$ & $1.289(2)$ \\
$\mathrm{C}(13)-\mathrm{N}(15)$ & $1.300(2)$ & $\mathrm{N}(15)-\mathrm{H}(14 \mathrm{~A})$ & 0.8600 \\
$\mathrm{~N}(14)-\mathrm{H}(14 \mathrm{~B})$ & 0.8600 & & 0.8600 \\
$\mathrm{~N}(15)-\mathrm{H}(15 \mathrm{~B})$ & 0.8600 & $\mathrm{~N}(3)-\mathrm{N}(2)-\mathrm{N}(1)$ & $110.05(14)$ \\
& & $\mathrm{C}(5)-\mathrm{N}(4)-\mathrm{N}(3)$ & $104.29(15)$ \\
$\mathrm{C}(5)-\mathrm{N}(1)-\mathrm{N}(2)$ & $103.62(15)$ & $\mathrm{N}(4)-\mathrm{C}(5)-\mathrm{C}(6)$ & $123.88(16)$ \\
$\mathrm{N}(2)-\mathrm{N}(3)-\mathrm{N}(4)$ & $109.27(15)$ & $\mathrm{C}(5)-\mathrm{C}(6)-\mathrm{N}(7)$ & $106.46(14)$ \\
$\mathrm{N}(4)-\mathrm{C}(5)-\mathrm{N}(1)$ & $112.78(15)$ & $\mathrm{N}(7)-\mathrm{C}(6)-\mathrm{C}(13)$ & $110.97(13)$ \\
$\mathrm{N}(1)-\mathrm{C}(5)-\mathrm{C}(6)$ & $123.31(16)$ & $\mathrm{N}(7)-\mathrm{C}(6)-\mathrm{N}(10)$ & $106.59(13)$ \\
$\mathrm{C}(5)-\mathrm{C}(6)-\mathrm{C}(13)$ & $116.83(14)$ & $\mathrm{O}(8)-\mathrm{N}(7)-\mathrm{O}(9)$ & $128.01(17)$ \\
$\mathrm{C}(5)-\mathrm{C}(6)-\mathrm{N}(10)$ & $110.00(13)$ & $\mathrm{O}(9)-\mathrm{N}(7)-\mathrm{C}(6)$ & $116.81(16)$ \\
$\mathrm{C}(13)-\mathrm{C}(6)-\mathrm{N}(10)$ & $105.59(13)$ & $\mathrm{O}(12)-\mathrm{N}(10)-\mathrm{C}(6)$ & $116.43(16)$ \\
$\mathrm{O}(8)-\mathrm{N}(7)-\mathrm{C}(6)$ & $115.04(15)$ & $\mathrm{N}(14)-\mathrm{C}(13)-\mathrm{N}(15)$ & $123.54(16)$ \\
$\mathrm{O}(12)-\mathrm{N}(10)-\mathrm{O}(11)$ & $127.60(18)$ & $\mathrm{N}(15)-\mathrm{C}(13)-\mathrm{C}(6)$ & $118.89(16)$ \\
$\mathrm{O}(11)-\mathrm{N}(10)-\mathrm{C}(6)$ & $115.87(15)$ & $\mathrm{C}(13)-\mathrm{N}(14)-\mathrm{H}(14 \mathrm{~B})$ & 120.0 \\
$\mathrm{~N}(14)-\mathrm{C}(13)-\mathrm{C}(6)$ & $117.45(15)$ & $\mathrm{C}(13)-\mathrm{N}(15)-\mathrm{H}(15 \mathrm{~A})$ & 120.0 \\
$\mathrm{C}(13)-\mathrm{N}(14)-\mathrm{H}(14 \mathrm{~A})$ & 120.0 & $\mathrm{H}(15 \mathrm{~A})-\mathrm{N}(15)-\mathrm{H}(15 \mathrm{~B})$ & 120.0 \\
$\mathrm{H}(14 \mathrm{~A})-\mathrm{N}(14)-\mathrm{H}(14 \mathrm{~B})$ & 120.0 & & \\
$\mathrm{C}(13)-\mathrm{N}(15)-\mathrm{H}(15 \mathrm{~B})$ & 120.0 & &
\end{tabular}


Table S19. Torsion angles $\left[{ }^{\circ}\right]$ for 10.

\begin{tabular}{lc}
\hline $\mathrm{C}(5)-\mathrm{N}(1)-\mathrm{N}(2)-\mathrm{N}(3)$ & $-0.2(2)$ \\
$\mathrm{N}(1)-\mathrm{N}(2)-\mathrm{N}(3)-\mathrm{N}(4)$ & $0.3(2)$ \\
$\mathrm{N}(2)-\mathrm{N}(3)-\mathrm{N}(4)-\mathrm{C}(5)$ & $-0.2(2)$ \\
$\mathrm{N}(3)-\mathrm{N}(4)-\mathrm{C}(5)-\mathrm{N}(1)$ & $0.1(2)$ \\
$\mathrm{N}(3)-\mathrm{N}(4)-\mathrm{C}(5)-\mathrm{C}(6)$ & $177.94(16)$ \\
$\mathrm{N}(2)-\mathrm{N}(1)-\mathrm{C}(5)-\mathrm{N}(4)$ & $0.1(2)$ \\
$\mathrm{N}(2)-\mathrm{N}(1)-\mathrm{C}(5)-\mathrm{C}(6)$ & $-177.80(16)$ \\
$\mathrm{N}(4)-\mathrm{C}(5)-\mathrm{C}(6)-\mathrm{N}(7)$ & $155.81(17)$ \\
$\mathrm{N}(1)-\mathrm{C}(5)-\mathrm{C}(6)-\mathrm{N}(7)$ & $-26.6(2)$ \\
$\mathrm{N}(4)-\mathrm{C}(5)-\mathrm{C}(6)-\mathrm{C}(13)$ & $31.2(3)$ \\
$\mathrm{N}(1)-\mathrm{C}(5)-\mathrm{C}(6)-\mathrm{C}(13)$ & $-151.14(17)$ \\
$\mathrm{N}(4)-\mathrm{C}(5)-\mathrm{C}(6)-\mathrm{N}(10)$ & $-89.1(2)$ \\
$\mathrm{N}(1)-\mathrm{C}(5)-\mathrm{C}(6)-\mathrm{N}(10)$ & $88.6(2)$ \\
$\mathrm{C}(5)-\mathrm{C}(6)-\mathrm{N}(7)-\mathrm{O}(8)$ & $-84.28(18)$ \\
$\mathrm{C}(13)-\mathrm{C}(6)-\mathrm{N}(7)-\mathrm{O}(8)$ & $43.8(2)$ \\
$\mathrm{N}(10)-\mathrm{C}(6)-\mathrm{N}(7)-\mathrm{O}(8)$ & $158.33(14)$ \\
$\mathrm{C}(5)-\mathrm{C}(6)-\mathrm{N}(7)-\mathrm{O}(9)$ & $91.73(18)$ \\
$\mathrm{C}(13)-\mathrm{C}(6)-\mathrm{N}(7)-\mathrm{O}(9)$ & $-140.15(16)$ \\
$\mathrm{N}(10)-\mathrm{C}(6)-\mathrm{N}(7)-\mathrm{O}(9)$ & $-25.66(19)$ \\
$\mathrm{C}(5)-\mathrm{C}(6)-\mathrm{N}(10)-\mathrm{O}(12)$ & $14.3(2)$ \\
$\mathrm{N}(7)-\mathrm{C}(6)-\mathrm{N}(10)-\mathrm{O}(12)$ & $129.29(19)$ \\
$\mathrm{C}(13)-\mathrm{C}(6)-\mathrm{N}(10)-\mathrm{O}(12)$ & $-112.62(19)$ \\
$\mathrm{C}(5)-\mathrm{C}(6)-\mathrm{N}(10)-\mathrm{O}(11)$ & $-169.01(17)$ \\
$\mathrm{N}(7)-\mathrm{C}(6)-\mathrm{N}(10)-\mathrm{O}(11)$ & $-53.99(19)$ \\
$\mathrm{C}(13)-\mathrm{C}(6)-\mathrm{N}(10)-\mathrm{O}(11)$ & $64.1(2)$ \\
$\mathrm{C}(5)-\mathrm{C}(6)-\mathrm{C}(13)-\mathrm{N}(14)$ & $-21.1(2)$ \\
$\mathrm{N}(7)-\mathrm{C}(6)-\mathrm{C}(13)-\mathrm{N}(14)$ & $-143.33(17)$ \\
$\mathrm{N}(10)-\mathrm{C}(6)-\mathrm{C}(13)-\mathrm{N}(14)$ & $101.56(18)$ \\
$\mathrm{C}(5)-\mathrm{C}(6)-\mathrm{C}(13)-\mathrm{N}(15)$ & $162.63(16)$ \\
$\mathrm{N}(7)-\mathrm{C}(6)-\mathrm{C}(13)-\mathrm{N}(15)$ & $40.4(2)$ \\
$\mathrm{N}(10)-\mathrm{C}(6)-\mathrm{C}(13)-\mathrm{N}(15)$ & $-74.75(19)$ \\
\hline &
\end{tabular}


Table S20. Hydrogen bonds for 10 [ $\left.\AA^{\text {and }}{ }^{\circ}\right]$.

\begin{tabular}{lcccc}
\hline \multicolumn{1}{c}{ D-H...A } & $\mathrm{d}(\mathrm{D}-\mathrm{H})$ & $\mathrm{d}(\mathrm{H} . . . \mathrm{A})$ & $\mathrm{d}(\mathrm{D} \ldots \mathrm{A})$ & $<(\mathrm{DHA})$ \\
\hline $\mathrm{N}(14)-\mathrm{H}(14 \mathrm{~A}) \ldots \mathrm{N}(2) \# 1$ & 0.86 & 1.99 & $2.830(2)$ & 165.5 \\
$\mathrm{~N}(14)-\mathrm{H}(14 \mathrm{~B}) \ldots \mathrm{N}(4)$ & 0.86 & 1.96 & $2.676(2)$ & 140.3 \\
$\mathrm{~N}(15)-\mathrm{H}(15 \mathrm{~B}) \ldots \mathrm{N}(3) \# 3$ & 0.86 & 2.06 & $2.901(2)$ & 165.4 \\
\hline
\end{tabular}

Symmetry transformations used to generate equivalent atoms:

\#1 x,y-1,z \#2 -x+1,-y,-z+2 \#3 x-1,y-1,z

Table S21. Crystal data and structure refinement for 12 .

\begin{tabular}{|c|c|c|}
\hline Identification code & \multicolumn{2}{|l|}{12} \\
\hline CCDC number & \multicolumn{2}{|l|}{1975701} \\
\hline Empirical formula & \multicolumn{2}{|l|}{$\mathrm{C}_{4} \mathrm{H}_{8} \mathrm{~N}_{8} \mathrm{O}_{5}$} \\
\hline Formula weight & \multicolumn{2}{|l|}{248.18} \\
\hline Temperature & \multicolumn{2}{|l|}{ 293(2) K } \\
\hline Wavelength & \multicolumn{2}{|l|}{$0.71073 \AA$} \\
\hline Crystal system & \multicolumn{2}{|l|}{ Monoclinic } \\
\hline Space group & \multicolumn{2}{|l|}{$\mathrm{P} 2{ }_{1} / \mathrm{c}$} \\
\hline Unit cell dimensions & $\begin{array}{l}a=4.9044(19) \AA \\
b=26.767(10) \AA \\
c=7.710(3) \AA\end{array}$ & $\begin{array}{l}\alpha=90^{\circ} . \\
\beta=96.835(12)^{\circ} . \\
\gamma=90^{\circ} .\end{array}$ \\
\hline Volume & \multicolumn{2}{|l|}{$1005.0(7) \AA^{3}$} \\
\hline $\mathrm{Z}$ & \multicolumn{2}{|l|}{4} \\
\hline Density $\left(20^{\circ} \mathrm{C}\right)$ & \multicolumn{2}{|l|}{$1.640 \mathrm{Mg} / \mathrm{m}^{3}$} \\
\hline $\begin{array}{l}\text { Absorption coefficient } \\
F(000)\end{array}$ & \multicolumn{2}{|l|}{$0.147 \mathrm{~mm}^{-1}$} \\
\hline Crystal size & \multicolumn{2}{|c|}{$0.261 \times 0.183 \times 0.020 \mathrm{~mm}^{3}$} \\
\hline Theta range for data collection & \multicolumn{2}{|l|}{1.522 to $25.614^{\circ}$} \\
\hline Index ranges & \multicolumn{2}{|c|}{$-5<=\mathrm{h}<=5,-32<=\mathrm{k}<=32,-9<=\mathrm{l}<=9$} \\
\hline Reflections collected & \multicolumn{2}{|l|}{6901} \\
\hline Independent reflections & \multicolumn{2}{|c|}{$1830\left[\mathrm{R}_{\mathrm{int}}=0.1204\right]$} \\
\hline Completeness to theta $=25.242^{\circ}$ & \multicolumn{2}{|c|}{$98.6 \%$} \\
\hline Absorption correction & \multicolumn{2}{|c|}{ Semi-empirical from equivalents } \\
\hline Max. and min. transmission & \multicolumn{2}{|c|}{0.7452 and 0.5251} \\
\hline $\begin{array}{l}\text { Refinement method } \\
\text { Data / restraints / parameters }\end{array}$ & \multicolumn{2}{|c|}{$\begin{array}{l}\text { Full-matrix least-squares on } \mathrm{F}^{2} \\
1830 / 0 / 155\end{array}$} \\
\hline Goodness-of-fit on $\mathrm{F}^{2}$ & \multicolumn{2}{|l|}{1.105} \\
\hline Final $R$ indices $[I>2 \operatorname{sigma}(I)]$ & \multicolumn{2}{|c|}{$\mathrm{R}_{1}=0.1169, \mathrm{wR}_{2}=0.2437$} \\
\hline $\mathrm{R}$ indices (all data) & \multicolumn{2}{|c|}{$\mathrm{R}_{1}=0.1947, \mathrm{wR}_{2}=0.2689$} \\
\hline Largest diff. peak and hole & \multicolumn{2}{|c|}{0.421 and -0.357 e. $\AA^{-3}$} \\
\hline
\end{tabular}


Table S22. Bond lengths $[\AA]$ and angles $\left[{ }^{\circ}\right]$ for $\mathbf{1 2}$.

\begin{tabular}{|c|c|c|c|}
\hline $\mathrm{O}(1)-\mathrm{N}(3)$ & $1.250(8)$ & $\mathrm{O}(2)-\mathrm{N}(3)$ & $1.234(8)$ \\
\hline $\mathrm{N}(3)-\mathrm{C}(4)$ & $1.388(9)$ & $\mathrm{C}(4)-\mathrm{N}(5)$ & $1.404(9)$ \\
\hline $\mathrm{C}(4)-\mathrm{C}(8)$ & $1.440(10)$ & $\mathrm{N}(5)-\mathrm{O}(6)$ & $1.233(8)$ \\
\hline $\mathrm{N}(5)-\mathrm{O}(7)$ & $1.240(8)$ & $\mathrm{C}(8)-\mathrm{N}(9)$ & $1.329(10)$ \\
\hline $\mathrm{C}(8)-\mathrm{N}(12)$ & $1.331(10)$ & $\mathrm{N}(9)-\mathrm{N}(10)$ & $1.336(9)$ \\
\hline $\mathrm{N}(9)-\mathrm{H}(9)$ & 0.8600 & $\mathrm{~N}(10)-\mathrm{N}(11)$ & $1.296(11)$ \\
\hline $\mathrm{N}(11)-\mathrm{N}(12)$ & $1.358(9)$ & $\mathrm{N}(13)-\mathrm{C}(15)$ & $1.314(10)$ \\
\hline $\mathrm{N}(13)-\mathrm{H}(13 \mathrm{~A})$ & 0.8600 & $\mathrm{~N}(13)-\mathrm{H}(13 \mathrm{~B})$ & 0.8600 \\
\hline $\mathrm{N}(14)-\mathrm{C}(15)$ & $1.295(10)$ & $\mathrm{N}(14)-\mathrm{H}(14 \mathrm{~A})$ & 0.8600 \\
\hline $\mathrm{N}(14)-\mathrm{H}(14 \mathrm{~B})$ & 0.8600 & $\mathrm{C}(15)-\mathrm{O}(16)$ & $1.296(9)$ \\
\hline $\mathrm{O}(16)-\mathrm{C}(17)$ & $1.451(9)$ & $\mathrm{C}(17)-\mathrm{H}(17 \mathrm{~A})$ & 0.9600 \\
\hline $\mathrm{C}(17)-\mathrm{H}(17 \mathrm{~B})$ & 0.9600 & $\mathrm{C}(17)-\mathrm{H}(17 \mathrm{C})$ & 0.9600 \\
\hline $\mathrm{O}(2)-\mathrm{N}(3)-\mathrm{O}(1)$ & 121.1(6) & $\mathrm{O}(2)-\mathrm{N}(3)-\mathrm{C}(4)$ & $122.8(6)$ \\
\hline $\mathrm{O}(1)-\mathrm{N}(3)-\mathrm{C}(4)$ & $116.1(6)$ & $\mathrm{N}(3)-\mathrm{C}(4)-\mathrm{N}(5)$ & $120.1(7)$ \\
\hline $\mathrm{N}(3)-\mathrm{C}(4)-\mathrm{C}(8)$ & $120.7(6)$ & $\mathrm{N}(5)-\mathrm{C}(4)-\mathrm{C}(8)$ & $119.1(6)$ \\
\hline $\mathrm{O}(6)-\mathrm{N}(5)-\mathrm{O}(7)$ & $120.5(6)$ & $\mathrm{O}(6)-\mathrm{N}(5)-\mathrm{C}(4)$ & $123.7(6)$ \\
\hline $\mathrm{O}(7)-\mathrm{N}(5)-\mathrm{C}(4)$ & $115.7(6)$ & $\mathrm{N}(9)-\mathrm{C}(8)-\mathrm{N}(12)$ & $107.2(7)$ \\
\hline $\mathrm{N}(9)-\mathrm{C}(8)-\mathrm{C}(4)$ & $125.9(7)$ & $\mathrm{N}(12)-\mathrm{C}(8)-\mathrm{C}(4)$ & $126.9(8)$ \\
\hline $\mathrm{C}(8)-\mathrm{N}(9)-\mathrm{N}(10)$ & $110.5(7)$ & $\mathrm{C}(8)-\mathrm{N}(9)-\mathrm{H}(9)$ & 124.8 \\
\hline $\mathrm{N}(10)-\mathrm{N}(9)-\mathrm{H}(9)$ & 124.8 & $\mathrm{~N}(11)-\mathrm{N}(10)-\mathrm{N}(9)$ & $105.4(7)$ \\
\hline $\mathrm{N}(10)-\mathrm{N}(11)-\mathrm{N}(12)$ & 111.1(6) & $\mathrm{C}(8)-\mathrm{N}(12)-\mathrm{N}(11)$ & $105.8(7)$ \\
\hline $\mathrm{C}(15)-\mathrm{N}(13)-\mathrm{H}(13 \mathrm{~A})$ & 120.0 & $\mathrm{C}(15)-\mathrm{N}(13)-\mathrm{H}(13 \mathrm{~B})$ & 120.0 \\
\hline $\mathrm{H}(13 \mathrm{~A})-\mathrm{N}(13)-\mathrm{H}(13 \mathrm{~B})$ & 120.0 & $\mathrm{C}(15)-\mathrm{N}(14)-\mathrm{H}(14 \mathrm{~A})$ & 120.0 \\
\hline $\mathrm{C}(15)-\mathrm{N}(14)-\mathrm{H}(14 \mathrm{~B})$ & 120.0 & $\mathrm{H}(14 \mathrm{~A})-\mathrm{N}(14)-\mathrm{H}(14 \mathrm{~B})$ & 120.0 \\
\hline $\mathrm{N}(14)-\mathrm{C}(15)-\mathrm{O}(16)$ & $123.5(7)$ & $\mathrm{N}(14)-\mathrm{C}(15)-\mathrm{N}(13)$ & $121.6(7)$ \\
\hline $\mathrm{O}(16)-\mathrm{C}(15)-\mathrm{N}(13)$ & $114.9(7)$ & $\mathrm{C}(15)-\mathrm{O}(16)-\mathrm{C}(17)$ & $119.4(6)$ \\
\hline $\mathrm{O}(16)-\mathrm{C}(17)-\mathrm{H}(17 \mathrm{~A})$ & 109.5 & $\mathrm{O}(16)-\mathrm{C}(17)-\mathrm{H}(17 \mathrm{~B})$ & 109.5 \\
\hline $\mathrm{H}(17 \mathrm{~A})-\mathrm{C}(17)-\mathrm{H}(17 \mathrm{~B})$ & 109.5 & $\mathrm{O}(16)-\mathrm{C}(17)-\mathrm{H}(17 \mathrm{C})$ & 109.5 \\
\hline $\mathrm{H}(17 \mathrm{~A})-\mathrm{C}(17)-\mathrm{H}(17 \mathrm{C})$ & 109.5 & $\mathrm{H}(17 \mathrm{~B})-\mathrm{C}(17)-\mathrm{H}(17 \mathrm{C})$ & 109.5 \\
\hline
\end{tabular}


Table S23. Torsion angles [ $\left.{ }^{\circ}\right]$ for 12.

\begin{tabular}{lc}
\hline $\mathrm{O}(2)-\mathrm{N}(3)-\mathrm{C}(4)-\mathrm{N}(5)$ & $-1.5(14)$ \\
$\mathrm{O}(1)-\mathrm{N}(3)-\mathrm{C}(4)-\mathrm{N}(5)$ & $178.7(8)$ \\
$\mathrm{O}(2)-\mathrm{N}(3)-\mathrm{C}(4)-\mathrm{C}(8)$ & $178.3(9)$ \\
$\mathrm{O}(1)-\mathrm{N}(3)-\mathrm{C}(4)-\mathrm{C}(8)$ & $-1.5(12)$ \\
$\mathrm{N}(3)-\mathrm{C}(4)-\mathrm{N}(5)-\mathrm{O}(6)$ & $-2.9(14)$ \\
$\mathrm{C}(8)-\mathrm{C}(4)-\mathrm{N}(5)-\mathrm{O}(6)$ & $177.3(8)$ \\
$\mathrm{N}(3)-\mathrm{C}(4)-\mathrm{N}(5)-\mathrm{O}(7)$ & $175.4(8)$ \\
$\mathrm{C}(8)-\mathrm{C}(4)-\mathrm{N}(5)-\mathrm{O}(7)$ & $-4.4(13)$ \\
$\mathrm{N}(3)-\mathrm{C}(4)-\mathrm{C}(8)-\mathrm{N}(9)$ & $139.4(9)$ \\
$\mathrm{N}(5)-\mathrm{C}(4)-\mathrm{C}(8)-\mathrm{N}(9)$ & $-40.8(13)$ \\
$\mathrm{N}(3)-\mathrm{C}(4)-\mathrm{C}(8)-\mathrm{N}(12)$ & $-39.7(13)$ \\
$\mathrm{N}(5)-\mathrm{C}(4)-\mathrm{C}(8)-\mathrm{N}(12)$ & $140.1(9)$ \\
$\mathrm{N}(12)-\mathrm{C}(8)-\mathrm{N}(9)-\mathrm{N}(10)$ & $0.7(10)$ \\
$\mathrm{C}(4)-\mathrm{C}(8)-\mathrm{N}(9)-\mathrm{N}(10)$ & $-178.6(8)$ \\
$\mathrm{C}(8)-\mathrm{N}(9)-\mathrm{N}(10)-\mathrm{N}(11)$ & $-0.3(10)$ \\
$\mathrm{N}(9)-\mathrm{N}(10)-\mathrm{N}(11)-\mathrm{N}(12)$ & $-0.2(11)$ \\
$\mathrm{N}(9)-\mathrm{C}(8)-\mathrm{N}(12)-\mathrm{N}(11)$ & $-0.8(10)$ \\
$\mathrm{C}(4)-\mathrm{C}(8)-\mathrm{N}(12)-\mathrm{N}(11)$ & $178.5(8)$ \\
$\mathrm{N}(10)-\mathrm{N}(11)-\mathrm{N}(12)-\mathrm{C}(8)$ & $0.6(11)$ \\
$\mathrm{N}(14)-\mathrm{C}(15)-\mathrm{O}(16)-\mathrm{C}(17)$ & $2.8(15)$ \\
$\mathrm{N}(13)-\mathrm{C}(15)-\mathrm{O}(16)-\mathrm{C}(17)$ & $-176.6(9)$ \\
\hline
\end{tabular}


Table S24. Hydrogen bonds for 12 [^ and $\left.{ }^{\circ}\right]$.

\begin{tabular}{lcccc}
\hline \multicolumn{1}{c}{ D-H...A } & $\mathrm{d}(\mathrm{D}-\mathrm{H})$ & $\mathrm{d}(\mathrm{H} \ldots \mathrm{A})$ & $\mathrm{d}(\mathrm{D} \ldots \mathrm{A})$ & $<(\mathrm{DHA})$ \\
\hline $\mathrm{N}(9)-\mathrm{H}(9) \ldots \mathrm{N}(12) \# 1$ & 0.86 & 1.96 & $2.766(10)$ & 155.0 \\
$\mathrm{~N}(14)-\mathrm{H}(14 \mathrm{~A}) \ldots \mathrm{O}(7) \# 2$ & 0.86 & 2.09 & $2.926(8)$ & 162.6 \\
$\mathrm{~N}(14)-\mathrm{H}(14 \mathrm{~B}) \ldots \mathrm{O}(2)$ & 0.86 & 2.11 & $2.889(9)$ & 150.7 \\
$\mathrm{~N}(13)-\mathrm{H}(13 \mathrm{~B}) \ldots \mathrm{O}(1) \# 3$ & 0.86 & 2.10 & $2.943(8)$ & 168.6 \\
$\mathrm{~N}(13)-\mathrm{H}(13 \mathrm{~A}) \ldots \mathrm{O}(6)$ & 0.86 & 2.10 & $2.850(9)$ & 144.6 \\
$\mathrm{~N}(13)-\mathrm{H}(13 \mathrm{~A}) \ldots \mathrm{O}(2)$ & 0.86 & 2.27 & $3.004(9)$ & 143.7 \\
\end{tabular}

Symmetry transformations used to generate equivalent atoms:

$\# 1 \mathrm{x}-1, \mathrm{y}, \mathrm{z} \quad \# 2 \mathrm{x}+1,-\mathrm{y}+3 / 2, \mathrm{z}+1 / 2 \quad \# 3 \mathrm{x}-1,-\mathrm{y}+3 / 2, \mathrm{z}-1 / 2$

\section{Covalent Bonding Interactions}

Table S25. Topological parameters of the bond critical points in FOX-7, 3, 4, 5 (BCPs, 3-1) from Multiwfn.

\begin{tabular}{|c|c|c|c|c|c|c|c|c|c|c|c|}
\hline Comp. & Bond & $\begin{array}{l}d_{\mathrm{BP}} \\
(\AA)^{[\mathrm{a}]}\end{array}$ & $\begin{array}{l}d 1_{\mathrm{BP}} \\
(\AA)^{[\mathrm{b}]} \\
\end{array}$ & $\begin{array}{l}d 2_{\mathrm{BP}} \\
(\AA)^{[\mathrm{c}]} \\
\end{array}$ & $\begin{array}{l}\rho \\
(\mathrm{au})^{[\mathrm{d}]}\end{array}$ & $\begin{array}{l}\nabla^{2} \rho \\
(\mathrm{rBCP})^{[\mathrm{e}]}\end{array}$ & $\lambda_{1}{ }^{[\mathrm{f}]}$ & $\lambda_{2}{ }^{[\mathrm{f}]}$ & $\lambda_{3}{ }^{[f]}$ & $\varepsilon^{[g]}$ & $\eta^{[\mathrm{h}]}$ \\
\hline \multirow{3}{*}{ FOX-7 } & $\mathrm{C} 5-\mathrm{C} 4$ & 1.421 & 0.734 & 0.686 & 0.293 & -0.759 & -0.630 & -0.464 & 0.336 & 0.357 & 1.875 \\
\hline & $\mathrm{C} 5-\mathrm{N} 9$ & 1.345 & 0.515 & 0.830 & 0.335 & -1.039 & -0.753 & -0.653 & 0.366 & 0.154 & 2.055 \\
\hline & $\mathrm{C} 4-\mathrm{N} 3$ & 1.438 & 0.549 & 0.889 & 0.277 & -0.712 & -0.609 & -0.422 & 0.319 & 0.442 & 1.907 \\
\hline \multirow{5}{*}{3} & $\mathrm{C} 5-\mathrm{C} 4$ & 1.435 & 0.750 & 0.685 & 0.287 & -0.742 & -0.611 & -0.469 & 0.338 & 0.303 & 1.807 \\
\hline & C5-N9 & 1.339 & 0.505 & 0.834 & 0.337 & -1.028 & -0.757 & -0.658 & 0.387 & 0.150 & 1.955 \\
\hline & C5-N6 & 1.339 & 0.507 & 0.832 & 0.337 & -1.030 & -0.756 & -0.656 & 0.383 & 0.303 & 1.807 \\
\hline & C4-N3 & 1.409 & 0.506 & 0.903 & 0.286 & -0.635 & -0.608 & -0.398 & 0.371 & 0.527 & 1.640 \\
\hline & $\mathrm{C} 4-\mathrm{C} 12$ & 1.449 & 0.706 & 0.743 & 0.277 & -0.703 & -0.577 & -0.467 & 0.340 & 0.236 & 1.694 \\
\hline \multirow{5}{*}{4} & $\mathrm{C} 5-\mathrm{C} 4$ & 1.435 & 0.752 & 0.683 & 0.287 & -0.742 & -0.611 & -0.468 & 0.337 & 0.305 & 1.813 \\
\hline & $\mathrm{C} 5-\mathrm{C} 9$ & 1.340 & 0.506 & 0.834 & 0.336 & -0.102 & -0.753 & -0.656 & 0.385 & 0.148 & 1.955 \\
\hline & C5-N6 & 1.340 & 0.507 & 0.833 & 0.337 & -0.103 & -0.755 & -0.655 & 0.382 & 0.152 & 1.979 \\
\hline & $\mathrm{C} 4-\mathrm{N} 3$ & 1.406 & 0.502 & 0.904 & 0.287 & -0.624 & -0.608 & -0.399 & 0.382 & 0.525 & 1.592 \\
\hline & C4-N12 & 1.456 & 1.717 & 0.739 & 0.275 & -0.691 & -0.573 & -0.464 & 0.345 & 0.235 & 1.658 \\
\hline \multirow{5}{*}{5} & C17-C18 & 1.420 & 0.747 & 0.673 & 0.293 & -0.764 & -0.626 & -0.467 & 0.329 & 0.340 & 1.901 \\
\hline & C17-N10 & 1.350 & 0.516 & 0.834 & 0.331 & -1.022 & -0.738 & -0.647 & 0.362 & 0.141 & 2.036 \\
\hline & C17-N13 & 1.351 & 0.518 & 0.832 & 0.332 & -1.030 & -0.741 & -0.648 & 0.358 & 0.143 & 2.068 \\
\hline & C18-N4 & 1.441 & 0.532 & 0.909 & 0.270 & -0.645 & -0.554 & -0.405 & 0.314 & 0.367 & 1.763 \\
\hline & $\mathrm{C} 18-\mathrm{C} 16$ & 1.460 & 0.725 & 0.735 & 0.277 & -0.704 & -0.585 & -0.475 & 0.357 & 0.232 & 1.641 \\
\hline
\end{tabular}

[a] $d_{\mathrm{BP}}$ the total length of the bond path (BP); [b] $d 1_{\mathrm{BP}}$ the distance of the first named atom to the $\mathrm{BCP}$; [c] $d 2_{\mathrm{BP}}$ the distance of the the BCP to the second named atom; [d] $\rho$ : the electron density; [e] $\nabla^{2} \rho:$ the Laplacian; [f] $\lambda_{1}, \lambda_{2}$ and $\lambda_{3}$ are the Hessian eigenvalues; $[\mathrm{g}] \varepsilon$ is the bond ellipticity $\left(\varepsilon=\lambda_{1} / \lambda_{2}-1\right) ;[\mathrm{h}] \eta=\left|\lambda_{1}\right| / \lambda_{3}$. 


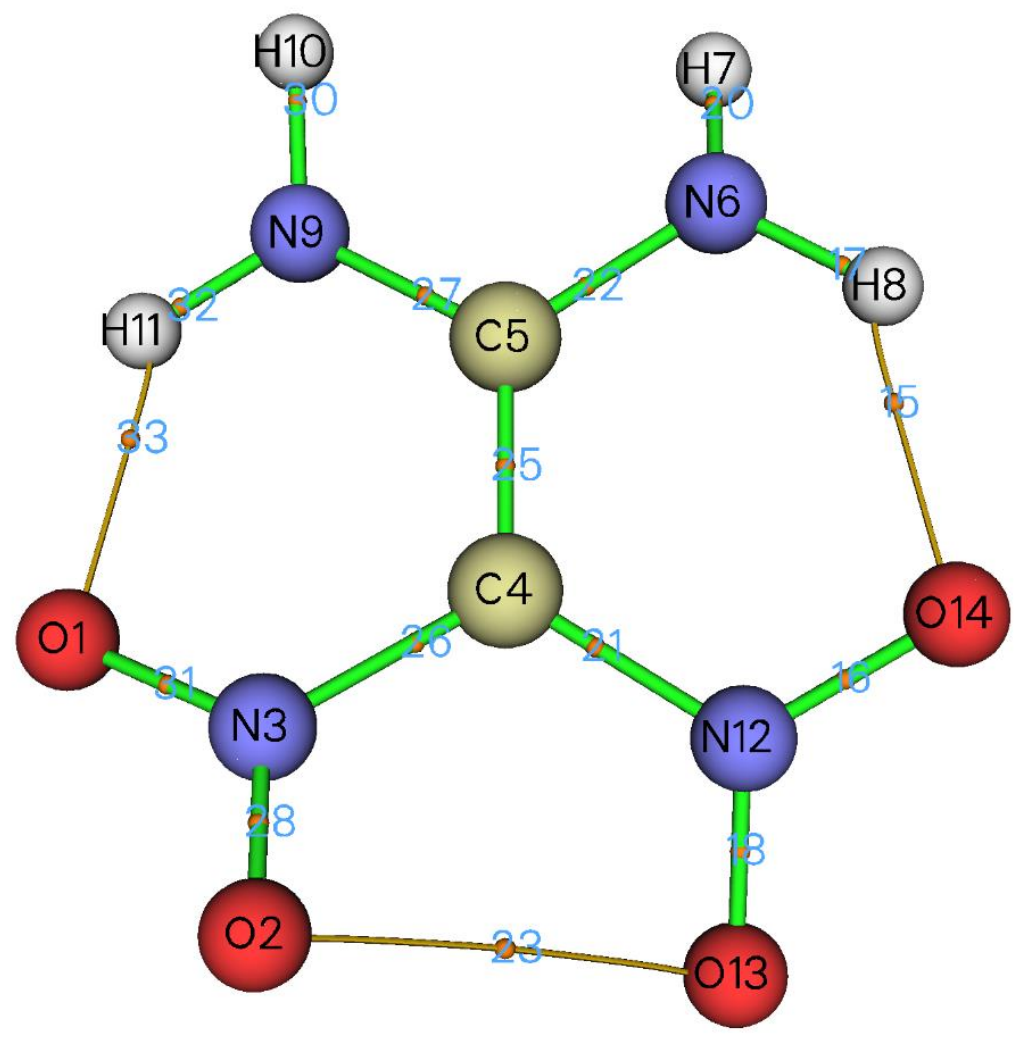

Figure S1. Molecular graph of FOX-7 depicting the bond paths (thinner, gold lines) and (3, 1) critical points (orange spheres)

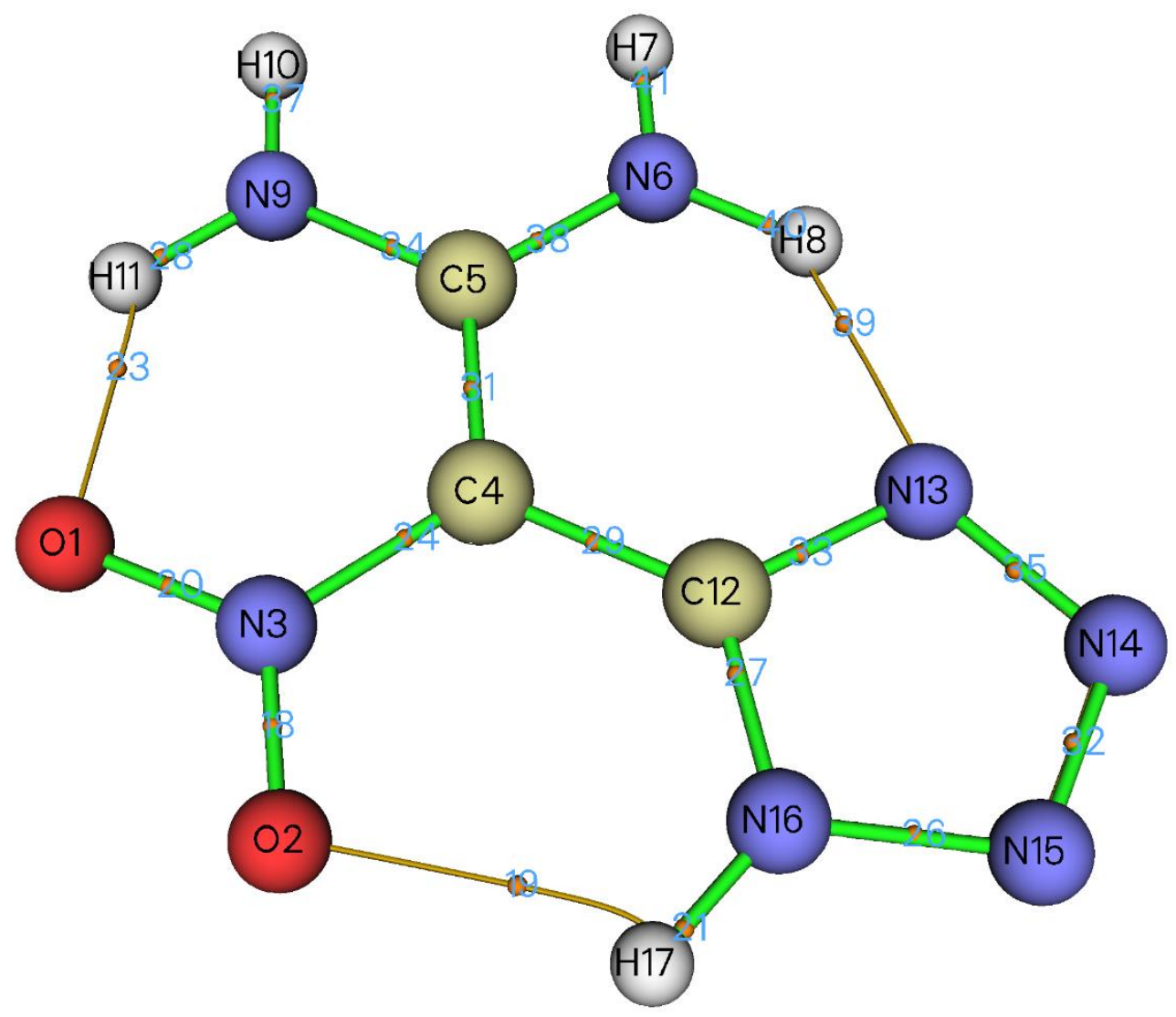

Figure S2. Molecular graph of $\mathbf{3}$ depicting the bond paths (thinner, gold lines) and (3, -1) critical points (orange spheres) 


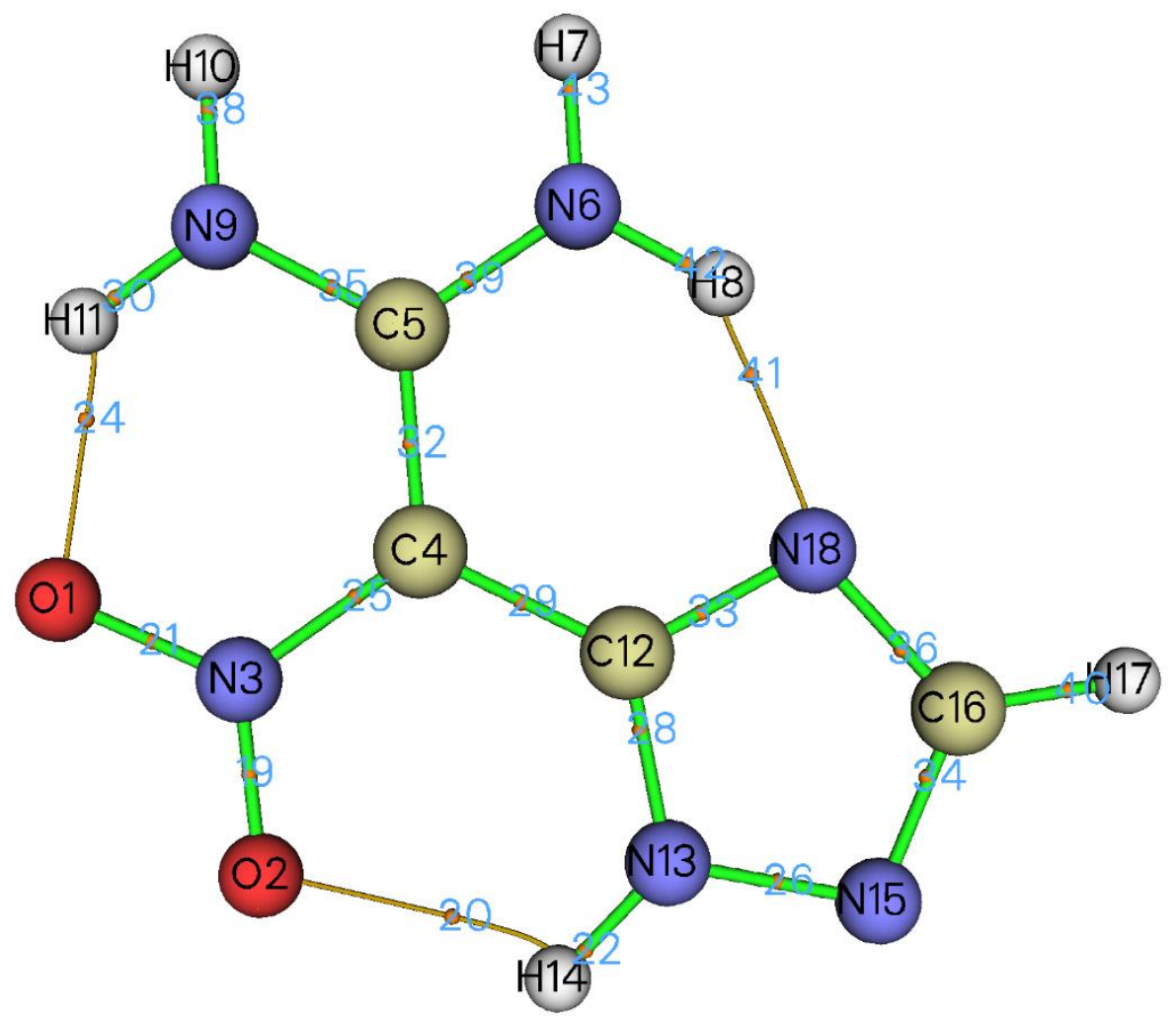

Figure S3. Molecular graph of 4 depicting the bond paths (thinner, gold lines) and (3, -1) critical points (orange spheres)

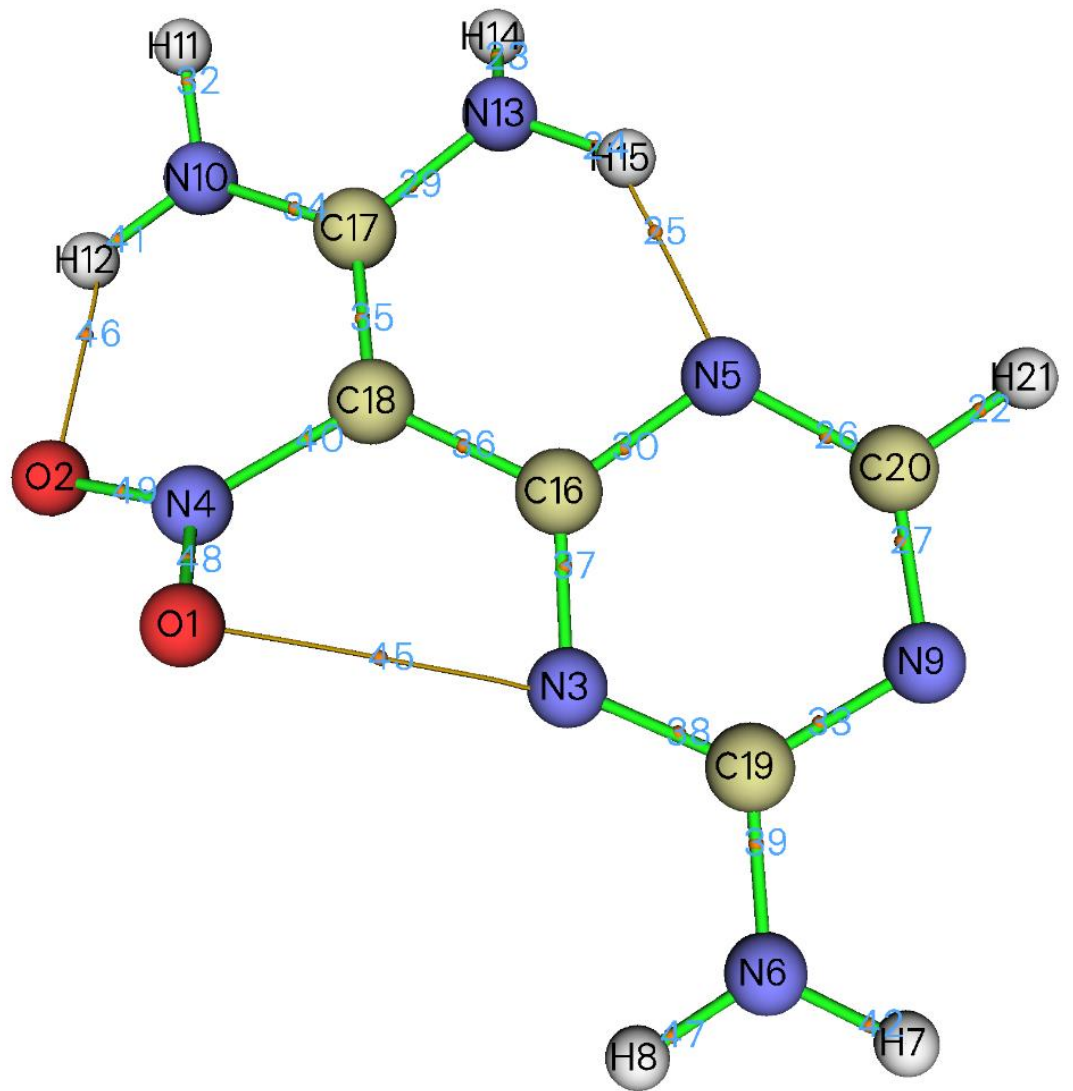

Figure S4. Molecular graph of 5 depicting the bond paths (thinner, gold lines) and (3, -1) critical points (orange spheres) 